\title{
Supersymmetry in a Space with Auxiliary Dimensions
}

\author{
A. A. Rosly and A. S. Schwarz \\ Institute of Theoretical and Experimental Physics, SU-117259 Moscow, USSR
}

\begin{abstract}
The purpose of this paper is to clarify the geometrical constructions leading to unconstrained superfields in extended supersymmetry.
\end{abstract}

\section{Introduction}

Recently great progress was made in constructing off-shell superfield theories in the case of extended supersymmetry. Galperin, Ivanov, Kalitzin, Ogievetsky, and Sokatchev succeeded in finding unconstrained superfield formulations for supersymmetric $N=2$ matter, supergravity, and $N=2$ and 3 Yang-Mills theories $[1,2]$. The crucial point that allowed them to overcome the $N=3$ barrier (for maximum spin one, and $N=2$ barrier for maximum spin one half) was the use of special superspaces with auxiliary variables. These superspaces emerged previously in an analog of Ward's twistor construction [3] which was suggested in [4] for the purpose of solving $N=2$ and $N=3$ superspace constraints. As a matter of fact, the description of $N=2,3$ unconstrained superfields and their relations with superfields in the usual Minkowski superspace can be made very close to the Ward construction (as well as to some other twistor constructions based on Ward's idea; see [5-7]). The purpose of the present paper is to clarify the geometrical origin of new unconstrained superfields introduced by Galperin et al. and to show how to come naturally to the results of $[1,2]$ starting from an analog of Ward transformation considered in [4]. This study has led us also to the construction of an unconstrained off-shell formulation for the general $N=2$ supersymmetric hyper-Kähler sigma model, which will be described elsewhere. (Interestingly enough, the treatment of the hyper-Kähler manifold proper to $N=2$ supersymmetry demonstrates once again a close analogy with twistor constructions - this time with a description of gravitational instantons due to Penrose [5].)

The key idea of introducing auxiliary dimensions borrowed from twistor constructions is based on the following simple observation. It turns out that if the extra dimensions added to some space form a compact complex manifold, then there is no actual increase of dimension for those fields, which are holomorphic with respect to these extra variables. This follows from the statement that any 
holomorphic function on a compact complex manifold is contant. (This can be derived, in turn, from the Liouville theorem which states that any bounded holomorphic function is constant.) The space $P$ with auxiliary dimensions relevant to extended supersymmetry will be just an example of that nature. This space was named by Galperin et al. the harmonic superspace [1, 2]. We will not use, however, the harmonic expansion with respect to extra variables on $P$ (which is still important if one is to obtain an off-shell component formulation in the usual Minkowski space ${ }^{1}$ with the group $\mathrm{SU}(N)$ realised linearly). Instead, we shall use geometrical constructions of [4]. Therefore, we prefer here to call the space $P$ the isotwistor space (for reasons that will be clear later). The superspace $P$ is related to the ordinary Minkowski superspace $M$ in a way analogous to the relation, for example, of $\mathbb{C} P^{3}$ to the Euclidean space-time $S^{4}$ in the original construction of Ward. (One is only to consider isospinors instead of spinors.) Note, however, that we will not be concerned with the questions global in $M$ (in particular, $M$ will not be compactified). Thus we shall deal with $P=M \times$ compact complex bosonic manifold $\}$ and our considerations will always be local with respect to coordinates in $M$. (For an appropriate global description of isotwistor space and its use see [14].) Nevertheless, it must be stressed that the treatment of auxiliary complex variables should be global (as is clear from the above remarks).

Let us review briefly the basic points of constructions that will be descirbed in detail in subsequent sections. Let

$$
\nabla_{\alpha i}=D_{\alpha i}+\mathscr{A}_{\alpha i}, \quad \nabla_{\dot{\alpha}}{ }^{i}=\bar{D}_{\dot{\alpha}}{ }^{i}+\mathscr{A}_{\dot{\alpha}}{ }^{i} \quad(\mathrm{i}=1, \ldots, N)
$$

be the gauge covariant derivatives in Minkowski superspace. The first step is to notice [4] that if we define the operators $\nabla_{\alpha}=p^{i} \nabla_{\alpha i}, \nabla_{\dot{\alpha}}=u_{i} \nabla_{\dot{\alpha}}^{i}$, then the usual $N=2$ and $N=3$ constraints $[8,6]$ on the superfields $\mathscr{A}_{\alpha i}, \mathscr{A}_{\alpha}^{i}$ are equivalent to the condition $^{2}$

$$
\left\{\nabla_{\alpha}, \nabla_{\beta}\right\}=\left\{\nabla_{\dot{\alpha}}, \nabla_{\dot{\beta}}\right\}=\left\{\nabla_{\alpha}, \nabla_{\dot{\beta}}\right\}=0,
$$

satisfied for arbitrary given values of complex numbers $p^{i}, u_{i}$, provided $p^{i} u_{i}=0$. This suggests, in analogy with Ward construction, to extend the usual Minkowski superspace $M$ by adding the parameters $p^{i}$ and $u_{i}$ taken (separately) as homogeneous coordinates. Thus we obtain a superspace $P=M \times Q$ (the isotwistor space) with auxiliary dimensions which correspond to a compact complex manifold $Q$. For $M=2$ we have $Q=\mathbb{C} P^{1}$, while for $N=3, Q$ is a three-dimensional complex submanifold in $\mathbb{C} P^{2} \times \mathbb{C} P^{2}$ defined by the equation $p^{i} u_{i}=0$. The operators $\nabla_{\alpha}, \nabla_{\dot{\alpha}}$ defined above can be considered as operators on $P$. (These operators act on functions which have definite degree of homogeneity with respect to homogeneous coordinates in $P$; they alter the degree of homogeneity of a function by one.) On this way one obtains a correspondence [4] between the gauge

\footnotetext{
${ }^{1}$ Note, that for theories beyond the $N=3$ (or $N=2$ ) barrier the corresponding manifestly supersymmetric component formulation necessarily involves an infinite number of gauge and/or auxiliary degrees of freedom; see $[1,2]$

${ }^{2}$ In the complexified Minkowski superspace this condition means that the field strength restricted to certain (0|4)-dimensional complex submanifolds vanishes. This is analogous to the vanishing of the self-dual field strength on the so-called $\alpha$-planes [3]
} 
fields satisfying the $N=2$, or $N=3$, constraints in $M$ and certain geometrical objects on the isotwistor superspace $P$. To describe these objects it is convenient to use the notion of partial gauge field. When one has a usual gauge field, one can gauge all derivatives, that is all first order differential operators. If instead one introduces gauging only for a part of differential operators, we will say that the partial gauge field is defined. In the case considered, one can reformulate the original constraint equations in $M$ as the condition of vanishing field strengths for certain partial gauge fields ${ }^{3}$ on $P$.

Let $z^{s}$ be the auxiliary variables in $P=M \times Q$ corresponding to complex coordinates in $Q$. (Thus $s=1,2,3$ for $N=3$ and $s=1$ for $N=2$.) To gauge the derivatives $\bar{\partial}_{s} \equiv \partial / \partial \bar{z}^{s}$ means to introduce the corresponding partial gauge field $\mathscr{A}_{s}$ on $P$ with an obvious transformation law that makes the operators $\bar{\partial}_{s}+\mathscr{A}_{s}$ gauge covariant. We shall see that the constrained gauge fields in $M$ correspond to partial gauge fields $\mathscr{A}_{s}$ on $P$ which satisfy a sort of chirality condition (i.e. the superfields $\mathscr{A}_{s}$ do not depend on some fermionic variables) along with the condition of vanishing field strength. The latter condition means that

$$
\bar{\partial}_{s} \mathscr{A}_{t}-\bar{\partial}_{t} \mathscr{A}_{s}+\left[\mathscr{A}_{s}, \mathscr{A}_{t}\right]=0 \text {. }
$$

To obtain this correspondence, consider partial gauge fields of zero field strength on $P$ related to gauging of differential operators $D_{\alpha}=p^{i} D_{\alpha i}, D_{\dot{\alpha}}=u_{i} \bar{D}_{\dot{\alpha}}{ }^{i}, \bar{\partial}_{s}$. From every constrained gauge field $\mathscr{A}_{\alpha i}, \mathscr{A}_{\dot{\alpha}}{ }^{i}$ on Minkowski superspace one can construct such a partial gauge field on $P$ by setting $\nabla_{\alpha}=D_{\alpha}+\mathscr{A}_{\alpha}, \nabla_{\dot{\alpha}}=D_{\dot{\alpha}}+\mathscr{A}_{\dot{\alpha}}, \nabla_{s}$ $=\bar{\partial}_{s}$ (that is $\left.\mathscr{A}_{\alpha}=p^{i} \mathscr{A}_{\alpha i}, \mathscr{A}_{\dot{\alpha}}=u_{i} \mathscr{A}_{\dot{\alpha}}^{i}, \mathscr{A}_{s}=0\right)$. Conversely, if a partial gauge field on $P, \nabla_{\alpha}=D_{\alpha}+\mathscr{A}_{\alpha}, \nabla_{\dot{\alpha}}=D_{\dot{\alpha}}+\mathscr{A}_{\dot{\alpha}}, \nabla_{s}=\bar{\partial}_{s}+\mathscr{A}_{s}$, has vanishing field strength, then generically it is gauge equivalent to a field with $\mathscr{A}_{s}=0$. In this particular gauge, the condition of vanishing field strength implies that $\bar{\partial}_{s} \mathscr{A}_{\alpha}=\bar{\partial}_{s} \mathscr{A}_{\dot{\alpha}}=0$. It follows from the last relations that $\mathscr{A}_{\alpha}=p^{i} \mathscr{A}_{\alpha i}(x, \theta, \bar{\theta})$ and $\mathscr{A}_{\dot{\alpha}}=u_{i} \mathscr{A}_{\dot{\alpha}}^{i}(x, \theta, \bar{\theta})$ for some functions $\mathscr{A}_{\alpha i}, \mathscr{A}_{\dot{\alpha}}^{i}$ depending only on $x, \theta, \bar{\theta}$. The remaining equations of vanishing field strength amount to (1.1). Consequently, the functions $\mathscr{A}_{\alpha i}, \mathscr{A}_{\alpha}^{i}$ correspond to a gauge field on Minkowski superspace satisfying the usual constraints. On the other hand, a partial gauge field $\mathscr{A}_{\alpha}, \mathscr{A}_{\dot{\alpha}}, \mathscr{A}_{s}$ having zero field strength in $P$ is gauge equivalent to a field with $\mathscr{A}_{\alpha}=\mathscr{A}_{\dot{\alpha}}=0$. In this gauge, we deal with components $\mathscr{A}_{s}$ which enter covariant derivatives $\nabla_{s}=\bar{\partial}_{s}+\mathscr{A}_{s}$ and satisfy (1.2) and $D_{\alpha} \mathscr{A}_{s}$ $=D_{\dot{\alpha}} \mathscr{A}_{s}=0$. The latter equations can be solved in a manner similar to the case of $N=1$ chirality constraints.

For $N=2$, the partial gauge field has only one component, because the complex dimension of $Q=\mathbb{C} P^{1}$ equals one. Hence Eq. (1.2) is trivially satisfied in this case and one gets the unconstrained superfield formulation for $N=2$ super Yang-Mills theory [1].

It must be pointed out that the Minkowski superspace constraints $[8,6]$ implicit in our discussion above are kinematical only for $N=2$, while for $N=3$ they are equivalent to the equation of motion. The crucial observation made by the authors of [2] is that the $N=3$ equations of motion rewritten in the form (1.2) can

${ }^{3}$ This interpretation of constraints was described in [4] in terms of so-called CR-bundles over $P$. In fact, partial gauge field gives us a well-known alternative description of such bundles, as will be explained in the present paper (see Sect. 10) 
be derived from a Lagrangian. This yields an off-shell formulation of $N=3$ theory in terms of superfields on the space $P$. The action which leads to Eqs. (1.2) is as follows

$$
S=\int d^{10 / 8} \mu \varepsilon^{s t u} \operatorname{tr}\left(\mathscr{A}_{s} \bar{\partial}_{t} \mathscr{A}_{u}+\frac{2}{3} \mathscr{A}_{s} \mathscr{A}_{t} \mathscr{A}_{u}\right)+\text { c.c }
$$

where $d^{10 / 8} \mu$ is an appropriate measure on the (10|12)-dimensional superspace $P=M \times Q$ which does not include integration over 4 of $\theta$ 's. Note, however, that the complex coordinates, $z^{s}$, used above cannot be introduced globally on the manifold $Q$. Thus one has to use either a number of coordinate patches, or the homogeneous coordinates $\left(p^{i}\right),\left(u_{i}\right)$ constrained by $p^{i} u_{i}=0$. The correct meaning of the measure $d \mu$ in (1.1) will be explained later in terms of homogeneous coordinates in Sect. 9. Note, finally, that the manner of how the gauge invariance is realized in $N=3$ Lagrangian (super) field theory and the very form of the action (1.2) are similar to the familiar topological mass (or Chern-Simons) term in threedimensional (ordinary) gauge theories [9].

The paper is organised as follows. In Sect. 2, we deal with a geometrical interpretation of constraints in $N=2$ super Yang-Mills superfield theory. This leads us to introduction of auxiliary variables. The basic superspace with auxiliary variables, in particular, the isotwistor space, relevant to the $N=2$ case are described in Sect. 3. In Sect. 4, a reformulation of $N=2$ super Yang-Mills constraints is constructed in terms of superfields on isotwistor space. The resulting unconstrained superfield formulation of this theory is described in Sect. 5. In Sect. 6 we show that the $N=2$ hypermultiplet can be dealt with in an analogous manner. Starting from the superfield equations of motion for a hypermultiplet in Minkowski superspace we construct a formulation of these equations in terms of superfields on isotwistor space. (Note that for the $N=2$ hypermultiplet the only superfields available in Minkowski superspace are on shell.) Then, in Sect. 7, an off-shell formulation of this theory is discussed following [1]. Sections 8 and 9 deal with the $N=3$ super Yang-Mills theory in a manner described briefly above. Section 10 contains some mathematical matter which explains the use of the language of holomorphic bundles and CR-bundles. (It is this language which is often used in various twistor constructions $[3,6,7]$ as well as considerations of [4].) After that we will be able to discuss on a more accurate level, in Sect. 11, certain non-perturbative features of new superfield theories. This discussion reveals, in particular, that the off-shell superfield formulation of $N=3$ super YangMills theory (in contrast to $N=2$ ) seems to be not equivalent (even on the mass shell, but beyond perturbation theory) to the usual Minkowski space formulation.

\section{Constraints in $\mathbf{N}=\mathbf{2}$ Super Yang-Mills Theory}

In the $N=2$ and $N=3$ super Yang-Mills theories the following constraints are used $[8,6]$ :

$$
\begin{gathered}
\left\{\nabla_{\alpha i}, \nabla_{\beta j}\right\}+\left\{\nabla_{\alpha j}, \nabla_{\beta i}\right\}=0, \\
\left\{\nabla_{\dot{\alpha}}^{i}, \nabla_{\dot{\beta}}^{j}\right\}+\left\{\nabla_{\dot{\alpha}}^{j}, \nabla_{\dot{\beta}}^{i}\right\}=0, \\
\left\{\nabla_{\alpha i}, \nabla_{\dot{\beta}}^{j}\right\}-\frac{1}{N} \delta_{j}^{i}\left\{\nabla_{\alpha k}, \nabla_{\dot{\beta}}^{k}\right\}=0 .
\end{gathered}
$$


Here $\nabla_{\alpha i}=D_{\alpha i}+\mathscr{A}_{\alpha i}, \nabla_{\dot{\alpha}}{ }^{i}=\bar{D}_{\dot{\alpha}}{ }^{i}+\mathscr{A}_{\dot{\alpha}}{ }^{i}$ denote the gauge covariant derivatives with the gauge field $\left(\mathscr{A}_{\alpha i}, \mathscr{A}_{\dot{\alpha}}{ }^{i}\right)$. The coordinates in Minkowski superspace will be denoted by $x^{a}, \theta^{\alpha i}, \bar{\theta}^{\alpha}{ }_{i}$ (where $\bar{\theta}^{\alpha}{ }_{i}$ is complex conjugated to $\theta^{\alpha i}$ and $a=1, \ldots, 4 ; \alpha=1,2$; $i=1, \ldots, N)$. As usual, $D_{\alpha i}$ are super covariant derivatives, $D_{\alpha i}=\partial / \partial \theta^{\alpha i}$ $+i \sigma_{\alpha \beta}^{a}{ }_{\hat{\beta}} \bar{\beta}_{i}{ }_{i} \partial / \partial x^{a}$, while $\bar{D}_{\dot{\alpha}}{ }^{i}$ are their conjugates. Our aim is to solve the constraints (2.1) for $N=2$. (Note that for $N=2$ these constraints are kinematical.) At first we represent these constraints in a different form. Namely, we introduce the operators

$$
\begin{aligned}
& \nabla_{\alpha}=p^{i} \nabla_{\alpha i}=D_{\alpha}+\mathscr{A}_{\alpha}, \\
& \nabla_{\dot{\alpha}}=p_{i} \nabla_{\dot{\alpha}}^{i}=D_{\dot{\alpha}}+\mathscr{A}_{\dot{\alpha}},
\end{aligned}
$$

where $p^{i}$ are arbitrary complex numbers, $p_{i}=\varepsilon_{i j} p^{j}$, and

$$
\begin{aligned}
D_{\alpha} & =p^{i} D_{\alpha i}, \quad D_{\dot{\alpha}}=p_{i} \bar{D}_{\dot{\alpha}}{ }^{i}, \\
\mathscr{A}_{\alpha} & =p^{i} \mathscr{A}_{\alpha i}, \quad \mathscr{A}_{\dot{\alpha}}=p_{i} \mathscr{A}_{\dot{\alpha}}{ }^{i} .
\end{aligned}
$$

It is easy to check that constraints (2.1) are equivalent to the condition

$$
\left\{\nabla_{\alpha}, \nabla_{\beta}\right\}=\left\{\nabla_{\dot{\alpha}}, \nabla_{\dot{\beta}}\right\}=\left\{\nabla_{\alpha}, \nabla_{\dot{\beta}}\right\}=0,
$$

satisfied for every $\left(p^{1}, p^{2}\right)$. Geometrically the constraint (2.5) means that the field strength vanishes on the (0|4)-dimensional subspace spanned by the vectors (2.3). (Such tangent subspaces will be called isosubspaces.) This remark permits us to give a slightly different interpretation of constraints.

Let us consider the complexified Minkowski superspace $M^{c}$ with coordinates $x^{a}, \theta^{\alpha i}, \bar{\theta}^{\alpha}{ }_{i}$, where $x^{a}$ are now complex, and $\bar{\theta}^{\alpha}{ }_{i}$ may be not conjugated to $\theta^{\alpha i}$. The following surface of complex dimension $(0 \mid 4)$ can be defined in this superspace by means of the parametric equations,

$$
\begin{gathered}
x^{a}=y^{a}+i \varepsilon^{\alpha} p^{i} \sigma_{\alpha \beta}^{a} \eta^{\dot{\beta}}{ }_{i}-i \eta^{\alpha i} \sigma_{\alpha \beta}^{a} \varepsilon^{\dot{\beta}} p_{i}, \\
\theta^{\alpha i}=\eta^{\alpha i}+\varepsilon^{\alpha} p^{i}, \\
\bar{\theta}^{\dot{\alpha}}{ }_{i}=\eta^{\dot{\alpha}}{ }_{i}+\varepsilon^{\dot{\alpha}} p_{i} .
\end{gathered}
$$

Here $\varepsilon^{\alpha}, \varepsilon^{\dot{\alpha}}$ are independent complex (fermionic) parameters, while $y^{a}, \eta^{\alpha i}, \eta^{\dot{\alpha}}{ }_{i}$ and $p^{i}$ are arbitrary complex constants. The surfaces of the form (2.6) will be called isoplanes. It is easy to see that at every point of the surface (2.6) the tangent subspace is spanned by the vectors (2.3), i.e. every tangent space is an isosubspace. This means that the restriction of the field strength of a super gauge field satisfying (2.1) to the arbitrary isoplane vanishes. Conversely, the vanishing of the field strength on isoplanes implies the constraints (2.5) and, hence, (2.1).

It follows from (2.5) that for every $p^{1}, p^{2}$ the operators $\nabla_{\alpha}, \nabla_{\dot{\alpha}}$ can be represented in the pure gauge form

$$
\nabla_{\alpha}=g^{-1} D_{\alpha} g, \quad \nabla_{\dot{\alpha}}=g^{-1} D_{\dot{\alpha}} g,
$$

where the function $g$ taking values in the gauge group $G$ depends on parameters $p^{1}$, $p^{2}$ and variables $x, \theta, \bar{\theta}$. Let us note that when one replaces $p^{i}$ by $\lambda p^{i}$, the operators $\nabla_{\alpha}, \nabla_{\dot{\alpha}}$ and $D_{\alpha}, D_{\dot{\alpha}}$ are multiplied by the same complex number $\lambda$. We see that the transformation $p^{i} \rightarrow \lambda p^{i}$ does not violate (2.7) considered as an equation on $g$. Therefore the function $g$ can be chosen in such a way that it is invariant under the 
transformations $p^{i} \rightarrow \lambda p^{i}$ with $\lambda \neq 0$. This suggests that it is natural to consider $p^{1}, p^{2}$ as homogeneous coordinates. In other words, we assume that the coordinates $p^{i}$ and $\lambda p^{i}$ represent the same point. (We shall assume throughout that $\lambda \neq 0$ and $p^{1}$, $p^{2}$ do not vanish simultaneously.)

The space with homogeneous coordinates $p^{1}, p^{2}$ is called the complex projective line and is denoted by $\mathbb{C} P^{1}$. This space can be interpreted as the plane of a complex variable $z$ with a point at infinity added to it. In other words, $\mathbb{C} P^{1}$ is the Riemann sphere. (The point with homogeneous coordinates $p^{1}, p^{2}$ corresponds to the complex number $z=p^{1} / p^{2}$.)

\section{Basic Superspaces}

The considerations of the last section explain that it is natural to introduce superspaces which have the parameters $p^{1}, p^{2}$ as additional coordinates. (As it was mentioned above, these parameters must be considered as homogeneous coordinates.) The most important role for our aims will be played by the space $P$ obtained from Minkowski superspace $M$ by the addition of homogeneous coordinates $p^{i}$ to $x^{a}, \theta^{\alpha i}, \bar{\theta}^{\dot{\alpha}}{ }_{i}$. In other words, $P=M \times \mathbb{C} P^{1}$. As we have seen in Sect. 2 , the function $g$ in (2.7) can be considered as a function on $P$. Geometrically, the superspace $P$ can be interpreted as the manifold of all tangent isosubspaces at all points of $M$. We call the superspace $P$ the isotwistor space.

It is useful to consider also the superspace $\mathscr{F}=M^{c} \times \mathbb{C} P^{1}$. (To define $\mathscr{F}$ we replace $M$ by its complexification $M^{c}$ in the definition of $P$.) Finally, we define the superspace $\mathscr{P}$ as the manifold of all isoplanes [i.e. of all (0|4)-dimensional surfaces (2.6) in $\left.M^{c}\right]$. To introduce coordinates in $\mathscr{P}$ we observe that the equations of isoplane (2.6) can be rewritten in the following form:

$$
\begin{gathered}
x^{a}=y^{a}+i \varepsilon^{\alpha} \sigma_{\alpha \dot{\beta}}^{a} \Theta^{\beta}-i \Theta^{\alpha} \sigma_{\alpha \dot{\beta}}^{a} \varepsilon^{\dot{\beta}}, \\
p_{i} \theta^{\alpha i}=\Theta^{\alpha}, \quad \bar{p}_{i} \theta^{\alpha i}=|p|^{2} \varepsilon^{\alpha}, \\
p^{i} \bar{\theta}^{\dot{\alpha}}{ }_{i}=\Theta^{\dot{\alpha}}, \quad \bar{p}^{i} \bar{\theta}^{\dot{\alpha}}{ }_{i}=|p|^{2} \varepsilon^{\dot{\alpha}},
\end{gathered}
$$

where $y^{a}, \Theta^{\alpha}, \Theta^{\dot{\alpha}}, p^{i}$ are constants that fix an isoplane uniquely. (Here $\bar{p}_{i} \equiv\left(p^{i}\right)^{*}, \bar{p}^{i}$ $\equiv\left(p_{i}\right)^{*}=\varepsilon^{i j} \tilde{p}_{j}$, and $|p|^{2} \equiv p^{i} \bar{p}_{i} \neq 0$.) This means that one can consider $y^{a}, \Theta^{\alpha}, \Theta^{\dot{\alpha}}, p^{i}$ as coordinates in the space of all isoplanes, $\mathscr{P}$. One must take into account though that $p^{i}, \Theta^{\alpha}, \Theta^{\dot{\alpha}}$ multiplied by a common number still correspond to the same isoplane. Thus one must treat $p^{i}, \Theta^{\alpha}, \Theta^{\dot{\alpha}}$ as homogeneous coordinates (while $y^{a}$ are usual coordinates).

Let us point out that there is one and only one isoplane that passes through a given point in complex Minkowski superspace and which is tangent to a given isosubspace at that point. This implies that for every point of the space $\mathscr{F}$ (which is the space of all isosubspaces) there is a corresponding point in $\mathscr{P}$. The relation between the spaces $\mathscr{F}$ and $\mathscr{P}$ can be clarified using a coordinate, change in $\mathscr{F}$. Let us pass from the coordinates $x^{a}, \theta^{\alpha i}, \bar{\theta}^{\dot{\alpha}}{ }_{i}, p^{i}$ in $\mathscr{F}=M^{c} \times \mathbb{C} P^{1}$ to the following ones:

$$
\begin{gathered}
\Theta^{\alpha}=p_{i} \theta^{\alpha i}, \quad \Theta^{\dot{\alpha}}=p^{i} \bar{\theta}^{\dot{\alpha}}, \\
\widetilde{\Theta}^{\alpha}=|p|^{-2} \bar{p}_{i} \theta^{\alpha i}, \quad \widetilde{\Theta}^{\dot{\alpha}}=|p|^{-2} \bar{p}^{i} \bar{\theta}^{\dot{\alpha}}{ }_{i}, \\
y^{a}=x^{a}+i \Theta^{\alpha} \sigma_{\alpha \dot{\beta}}^{a} \widetilde{\Theta}^{\dot{\beta}}-i \widetilde{\Theta}^{\alpha} \sigma_{\alpha \dot{\beta}}^{a} \Theta^{\dot{\beta}} .
\end{gathered}
$$


Now the projection of $\mathscr{F}$ onto $\mathscr{P}$ described above can be given by mapping a point $\left(y^{a}, \Theta^{\alpha}, \Theta^{\dot{\alpha}}, \widetilde{\Theta}^{\alpha}, \widetilde{\Theta}^{\dot{\alpha}}, p^{i}\right)$ in $\mathscr{F}$ to the point with coordinates $\left(y^{a}, \Theta^{\alpha}, \Theta^{\dot{\alpha}}, p^{i}\right)$ in $\mathscr{P}$. [This can be checked using (3.1) and (3.2).]

It is obvious from our definitions that the space $P$ can be considered as a submanifold in $\mathscr{F}$. In coordinates $x^{a}, \theta^{\alpha i}, \bar{\theta}^{\alpha}, p^{i}$ this submanifold can be singled out by means of reality conditions: $x^{a}=\left(x^{a}\right)^{*}, \bar{\theta}^{\alpha}{ }_{i}=\left(\theta^{\alpha i}\right)^{*}$, while in coordinates (3.2) this can be expressed as

$$
\begin{gathered}
\Theta^{\dot{\alpha}}=|p|^{2}\left(\tilde{\Theta}^{\alpha}\right)^{*}, \quad \tilde{\Theta}^{\dot{\alpha}}=|p|^{-2}\left(\Theta^{\alpha}\right)^{*}, \\
y^{a}-\left(y^{a}\right)^{*}=2 i \Theta^{\alpha} \sigma_{\alpha \dot{\beta}}^{a} \widetilde{\Theta}^{\dot{\beta}}-2 i \widetilde{\Theta}^{\alpha} \sigma_{\alpha \dot{\beta}}^{a} \Theta^{\dot{\beta}} .
\end{gathered}
$$

On the other hand, the space $P$ can be thought of also as being embedded in $\mathscr{P}$. Indeed, every point $\left(x^{a}, \theta^{\alpha i}, \bar{\theta}^{\dot{\alpha}}, p^{i}, \bar{p}_{i}\right)$ of $P$ can be considered as a point in $\mathscr{F}$ and, hence, there is a point in $\mathscr{P}$ corresponding to it with coordinates given by $(3.2 \mathrm{a}, \mathrm{c})$. It follows from the relations $(3.2 \mathrm{a}, \mathrm{c})$ that different points of $P$ are mapped to different points in $\mathscr{P}^{4}$, thus giving an embedding of $P$ into $\mathscr{P}$.

We proceed now to a discussion of various functions and differential operators on the isotwistor space $P$ that will be of most importance for us. First of all, let us point out that a function defined on $P$ can be considered in terms of coordinates $x^{a}$, $\theta^{\alpha i}, \bar{\theta}^{\alpha}{ }_{i}, p^{i}, \bar{p}_{i}$ as a function of these variables subjected to the requirement of homogeneity of degree zero (i.e. it must remain unchanged when $p^{i} \rightarrow \lambda p^{i}$ ). It will be useful also to deal with functions of $x^{a}, \theta^{\alpha i}, \bar{\theta}^{\dot{\alpha}}, p^{i}, \bar{p}_{i}$, which are homogeneous of degree $n$, that is they satisfy by definition

$$
f(x, \theta, \bar{\theta}, \lambda p, \bar{\lambda} \bar{p})=\lambda^{n} f(x, \theta, \bar{\theta}, p, \bar{p}) .
$$

Such functions will be called homogeneous (of degree $n$ ) functions on $P^{5}$.

Now the differential operators $D_{\alpha}$ and $D_{\dot{\alpha}}$ introduced earlier [see, (2.3)] can be considered as acting on functions on $P$ and transforming a homogeneous function of degree $n$ to a function of degree $n+1$. An important role will be played also by the operator $D$, which is defined by the formula,

$$
D=|p|^{2} p_{i} \frac{\partial}{\partial \bar{p}_{i}} .
$$

This operator increases the degree of homogeneity of a function by two. The operator $D$ is intimately connected with the well known operator $\bar{\partial}$, which acts on differential forms on $\mathbb{C} P^{1}$. [Applied, for example, to a function $f(p, \bar{p})$ on $\mathbb{C} P^{1}$, it gives a 1 -form which can be expressed in terms of homogeneous coordinates as follows: $\bar{\partial} f \equiv\left(\partial f / \partial \bar{p}_{i}\right) d \bar{p}_{i}=(D f) \cdot \omega$, where $\left.\omega=|p|^{-4} \bar{p}^{i} d \bar{p}_{i} \equiv|p|^{-4} \varepsilon^{i j} \bar{p}_{j} d \bar{p}_{i} \cdot\right]$

In what follows we shall have to deal with (complex) functions on $P$, subjected to the following conditions:

$$
D_{\alpha} \varphi=0, \quad D_{\dot{\alpha}} \varphi=0 .
$$

\footnotetext{
${ }^{4}$ Geometrically, this means that an isoplane of the form (3.1) in $M^{c}$ intersects the real Minkowski superspace $M$ in at most one point

${ }^{5}$ Note that homogeneous functions of two variables $p^{1}, p^{2}$ are in one-to-one correspondence with certain functions of one variable $z=p^{1} / p^{2}: f\left(p^{1}, p^{2}, \bar{p}_{1}, \bar{p}_{2}\right)=\left(p^{2}\right)^{n} f\left(p^{1} / p^{2}, 1, \bar{p}_{1} / \bar{p}_{2}, 1\right)$ $=\left(p^{2}\right)^{n} g(z, \bar{z})$, where $g(z, \bar{z}) \sim z^{n}$ as $z \rightarrow \infty$, and such asymptotic behaviour of $g(z, \bar{z})$ defines the class of functions of one variable that correspond to homogeneous functions of two variables
} 
Such functions will be called isochiral functions. It is essential that Eqs. (3.4) can be solved explicitly. (The situation is quite familiar from $N=1$ chiral superfields.) In order to describe the solution of (3.4) let us continue analytically the function $\varphi$ with respect to the variables corresponding to Minkowski superspace. Then we obtain a function on (a domain of ${ }^{6}$ the space $\mathscr{F}$ which is holomorphic in the variables $x^{a}, \theta^{\alpha i}, \bar{\theta}^{\dot{\alpha}}{ }_{i}$. It can be easily seen that Eqs. (3.4) expressed in terms of coordinates $y^{a}, \Theta^{\alpha}, \Theta^{\dot{\alpha}}, \widetilde{\Theta}^{\alpha}, \widetilde{\Theta}^{\dot{\alpha}}, p^{i}$ on $\mathscr{F}$ become

$$
\frac{\partial}{\partial \widetilde{\Theta}^{\alpha}} \varphi=0, \quad \frac{\partial}{\partial \widetilde{\Theta}^{\dot{\alpha}}} \varphi=0 .
$$

This means that an isochiral function continued analytically to the space $\mathscr{F}$ yields a function there which is holomorphic in $y^{a}, \Theta^{\alpha}, \Theta^{\dot{\alpha}}$, but does not depend on $\widetilde{\Theta}^{\alpha}$, $\widetilde{\Theta}^{\dot{\alpha}}$. It is seen from the connection between the spaces $\mathscr{F}$ and $\mathscr{P}$ described above, that the functions on $\mathscr{F}$ which do not depend on $\widetilde{\Theta}^{\alpha}, \widetilde{\Theta}^{\dot{\alpha}}$ can be considered as functions defined on $\mathscr{P}$. We obtain thus a correspondence between isochiral functions on $P$ and those functions on $\mathscr{P}$, which are holomorphic in $y^{a}, \Theta^{\alpha}, \Theta^{\dot{\alpha}}$.

Till now we did not consider conditions which might restrict the dependence of funtions on the complex variable corresponding to homogeneous coordinates $p^{1}$, $p^{2}$. Let us consider the solution of the equation

$$
D \varphi=0
$$

on the space $P$. [The operator $D$ was defined in (3.3).] It is easy to see that Eq. (3.6) amounts to the condition that the function is holomorphic with respect to homogeneous coordinates $p^{1}, p^{2}$. It is well known (and can be proved using the Liouville theorem), that every function defined for all values of the variables $p^{1}, p^{2}$, except $p^{1}=p^{2}=0$, and depending on them holomorphically and homogeneously of degree $n$ is equal to a polynomial of order $n$ in $p^{1}, p^{2}$, (In particular, there are no holomorphic functions which are homogeneous of degree $n<0$.) Thus, for instance, the solution to (3.6) with $n=1$ is simply an arbitrary linear function of $p^{1}$, $p^{2}$ if it is to be defined for all $p^{1}, p^{2},|p|^{2} \neq 0$.

\section{Conversion of $N=2$ Super Yang-Mills Theory to a Theory in Superspace with Auxiliary Dimensions}

We shall show here how to convert the super Yang-Mills theory to a field theory in the isotwistor space $P$. For this purpose we need a generalisation of the notion of a gauge field. Commonly, to introduce a gauge field means to define covariant derivatives. [That is to say, one has to define instead of the usual derivatives, say $\partial_{\mu}$ $=\partial / \partial x^{\mu}$, the covariant ones, $\nabla_{\mu}=\partial_{\mu}+\mathscr{A}_{\mu}(x)$.] One can gauge not only the derivatives with respect to coordinates, but also any first order differential operator $X=a^{\mu}(x) \partial_{\mu}$. (Gauging $X$ gives $\nabla_{X}=a^{\mu}(x)\left[\partial_{\mu}+\mathscr{A}_{\mu}(x)\right]$.) It will be convenient, for our purposes, to consider a kind of partial gauge field, that is we shall deal with a situation, when one gauges not all differential operators but only a

${ }^{6}$ Our considerations are always local with respect to coordinates of (possibly complexified) Minkowski superspace, although this is not always mentioned explicitly 
part of them. In fact, it will be useful to assume that in the space $P$ only the operators $D_{\alpha}, D_{\dot{\alpha}}$ and $D$ are gauged. Thus we are to consider a partial gauge field $\left(\mathscr{A}_{\alpha}, \mathscr{A}_{\dot{\alpha}}, \mathscr{A}\right)$ in $P$, which corresponds to introducing gauge covariant operators $\nabla_{\alpha}=D_{\alpha}+\mathscr{A}_{\alpha}, \nabla_{\dot{\alpha}}=D_{\dot{\alpha}}+\mathscr{A}_{\dot{\alpha}}, \nabla=D+\mathscr{A}$. As discussed in Sect. 3, the operators $D_{\alpha}, D_{\dot{\alpha}}$ raise the degree of homogeneity of a function by 1 , while $D$ raises it by 2 . Hence we have to assume the functions $\mathscr{A}_{\alpha}, \mathscr{A}_{\dot{\alpha}}$ and $\mathscr{A}$ on $P$ (taking values, as usual, in the Lie algebra of the gauge group) to be homogeneous of degrees 1, 1 and 2 respectively.

We will say that a partial gauge field has vanishing strength if the covariant derivatives (anti)commute with each other. Thus the field strength of $\left(\mathscr{A}_{\alpha}, \mathscr{A}_{\dot{\alpha}}, \mathscr{A}\right)$ vanishes if

$$
\begin{gathered}
\left\{\nabla_{\alpha}, \nabla_{\beta}\right\}=\left\{\nabla_{\dot{\alpha}}, \nabla_{\dot{\beta}}\right\}=\left\{\nabla_{\alpha}, \nabla_{\dot{\beta}}\right\}=0, \\
{\left[\nabla, \nabla_{\alpha}\right]=\left[\nabla, \nabla_{\dot{\alpha}}\right]=0 .}
\end{gathered}
$$

The gauge equivalence of partial gauge fields is defined in the conventional manner: the field $\left(\mathscr{A}_{\alpha}, \mathscr{A}_{\alpha}, \mathscr{A}\right)$ is called gauge equivalent to $\left(\mathscr{A}_{\alpha}^{\prime}, \mathscr{A}_{\alpha}^{\prime}, \mathscr{A}^{\prime}\right)$ if the corresponding covariant derivatives are connected by

$$
\nabla_{\alpha}^{\prime}=\hat{g}^{-1} \nabla_{\alpha} \hat{g}, \quad \nabla_{\dot{\alpha}}^{\prime}=\hat{g}^{-1} \nabla_{\dot{\alpha}} \hat{g}, \quad \nabla^{\prime}=\hat{g}^{-1} \nabla \hat{g},
$$

where $\hat{g}$ denotes the operator of multiplication by a function $g$ taking values in the gauge group. As a matter of course, a (partial) gauge field which is gauge equivalent to zero (i.e. a pure gauge) has vanishing strength. In the case of partial gauge fields, however, the inverse is valid only locally ${ }^{7}$. We wish to emphasize here that although our considerations are always local with respect to coordinates corresponding to Minkowski space, the dependence on (homogeneous) coordinates $p^{1}, p^{2}$ corresponding to $\mathbb{C} P^{1}$ in $P=M \times \mathbb{C} P^{1}$ is to be considered globally.

Even in the case of a single operator $\nabla=D+\mathscr{A}$, the field strength of which vanishes identically, it is not always possible to find a gauge function defined globally with respect to $\mathbb{C} P^{1}$, such that $\nabla=\hat{g}^{-1} D \hat{g}$. For an Abelian gauge group such a function does, in fact, always exist. But in non-Abelian case, the equation

$$
g^{-1} D g=\mathscr{A}
$$

has a solution not for any given function $\mathscr{A}$. Nevertheless, it is true that generically this equation still has a solution. That is to say, if Eq. (4.3) is compatible for some field $\mathscr{A}$, it is also compatible for all fields sufficiently near to $\mathscr{A}$. In particular, Eq. (4.3) is compatible if $\mathscr{A}$ is sufficiently small. [This fact, well known in mathematics, can be proved using power expansions. See Sect. 11 for more details about the solution of (4.3).]

Let us note, however, that the operators $\nabla_{\alpha}=D_{\alpha}+\mathscr{A}_{\alpha}, \nabla_{\dot{\alpha}}=D_{\dot{\alpha}}+\mathscr{A}_{\dot{\alpha}}$, if they anticommute, can be gauge transformed to $D_{\alpha}, D_{\dot{\alpha}}$ [because, in this case, the variables $p^{1}, p^{2}$ enter only as parameters). The same statement (in terms of Minkowski superspace) was already pointed out in Sect. 2].

The main conclusion we will reach in this section is that instead of gauge fields in Minkowski superspace satisfying the constraints (2.1), one can use partial gauge

\footnotetext{
${ }^{7}$ Note that even for ordinary gauge fields on a non-simply connected manifold the vanishing of the field strength does not also imply that the field is a pure gauge. In what follows we shall be able, however, not to be concerned with such a situation
} 
fields $\left(\mathscr{A}_{\alpha}, \mathscr{A}_{\dot{\alpha}}, \mathscr{A}\right)$ on $P$, which have vanishing field strength and satisfy the condition of compatibility of Eq. (4.3). To be more precise, the gauge equivalence classes of fields on the Minkowski superspace $M$ are in one-to-one correspondence with gauge equivalence classes of partial gauge fields on $P$, provided all the abovementioned requirements are satisfied.

To prove this, let us note first of all that for every gauge field, $\left(\mathscr{A}_{\alpha i}, \mathscr{A}_{\dot{\alpha}}{ }^{i}\right)$ on $M$, one can construct a field on $P$, setting

$$
\begin{aligned}
& \mathscr{A}_{\alpha}=p^{i} \mathscr{A}_{\alpha i}\left(x^{b}, \theta^{\beta j}, \bar{\theta}^{\dot{\beta}}{ }_{j}\right), \\
& \mathscr{A}_{\dot{\alpha}}=p_{i} \mathscr{A}_{\dot{\alpha}}{ }^{i}\left(x^{b}, \theta^{\beta j}, \bar{\theta}^{\dot{\beta}}{ }_{j}\right), \\
& \mathscr{A}=0 .
\end{aligned}
$$

All the (anti)commutators of the covariant derivatives $\nabla_{\alpha}, \nabla_{\dot{\alpha}}, \nabla$, corresponding to (4.4), among themselves vanish if the field $\left(\mathscr{A}_{\alpha i}, \mathscr{A}_{\dot{\alpha}}{ }^{i}\right)$ satisfies on $P$ the constraints of the super Yang-Mills theory (2.1). [A part of this statement concerning $\nabla_{\alpha}, \nabla_{\dot{\alpha}}$ was already discussed in Sect. 2; now one has thus to check only the commutators with $\nabla=D$ using the definitions (2.2) and (3.3).]

Conversely, let some field $\left(\mathscr{A}_{\alpha}^{\prime}, \mathscr{A}_{\dot{\alpha}}^{\prime}, \mathscr{A}^{\prime}\right)$ with vanishing field strength be given in $P$ satisfying additionally the condition that Eq. (4.3) has a solution. Then we are able to make a gauge transformation giving a new field $\left(\mathscr{A}_{\alpha}, \mathscr{A}_{\dot{\alpha}}, \mathscr{A}\right)$, such that $\mathscr{A}=0$. The field strength of the transformed field still vanishes, of course; that is the operators $\nabla_{\alpha}, \nabla_{\dot{\alpha}}, \nabla=D$ (anti)commute. From $\left[D, \nabla_{\alpha}\right]=\left[D, \nabla_{\dot{\alpha}}\right]=0$ we find that in the new gauge $D \mathscr{A}_{\alpha}=D \mathscr{A}_{\dot{\alpha}}=0$. As discussed in Sect. 3, these equations imply that the functions $\mathscr{A}_{\alpha}, \mathscr{A}_{\dot{\alpha}}$ (which by definition are homogeneous of degree one) are holomorphic in $p^{1}, p^{2}$ and, hence, linear in these variables. Thus they are of the form (4.4) for some functions $\mathscr{A}_{\alpha i}, \mathscr{A}_{\dot{\alpha}}{ }^{i}$, which can be considered as fields defined on Minkowski superspace $M$. Exploiting further the condition (4.1), we find that, for the fields (4.4), it follows from (4.1a) (as it was in Sect. 2) that the gauge field ( $\mathscr{A}_{\alpha i}$, $\mathscr{A}_{\dot{\alpha}}{ }^{i}$ ) on $M$ satisfies the constraints (2.1). We have thus described how to come back from the gauge fields on $P$ to the fields on Minkowski superspace.

It remains only to point out the by now obvious fact that when passing from $P$ to $M$ and from $M$ to $P$ - in both cases - the gauge equivalent fields go into the gauge equivalent ones.

The arguments used above are complete if the gauge fields considered are assumed to be complex. If the gauge fields $\left(\mathscr{A}_{\alpha i}, \mathscr{A}_{\dot{\alpha}}{ }^{i}\right)$ on $M$ satisfy a reality condition, e.g. $\mathscr{A}_{\dot{\alpha}}{ }^{i}=-\left(\mathscr{A}_{\alpha i}\right)^{+}$for $\mathrm{SU}(n)$ as the gauge group, then the reality condition for the corresponding gauge field $\left(\mathscr{A}_{\alpha}, \mathscr{A}_{\dot{\alpha}}, \mathscr{A}\right)$ on $P$ is as follows:

$$
\mathscr{A}_{\dot{\alpha}}(\hat{p})=-\left[\mathscr{A}_{\alpha}(\varrho(\hat{p}))\right]^{+}, \quad \mathscr{A}(\hat{p})=-[\mathscr{A}(\varrho(\hat{p}))]^{+} .
$$

Here $\hat{p}=\left(x, \theta, \bar{\theta}, p^{i}\right)$ denotes a point in the space $P$, while the transformation $\varrho$ acts on $P$ as $\hat{p} \rightarrow \varrho(\hat{p})=\left(x, \theta, \bar{\theta}, \bar{p}^{i}\right)$, where according to our conventions $\bar{p}^{i}=\varepsilon^{i j} \bar{p}_{j}$ $=\varepsilon^{i j}\left(p^{j}\right)^{*}$.

\section{Unconstrained Superfield Formulation of $N=2$ Super Yang-Mills Theory}

In order to find such a formulation we will use the reduction of the usual constraints (2.1) to certain conditions on the fields in the space $P$, as described 
before. According to Sect. 4, from the gauge field subjected to (2.1) on Minkowski superspace, we can construct on $P$ a partial gauge field $\left(\mathscr{A}_{\alpha}, \mathscr{A}_{\dot{\alpha}}, \mathscr{A}\right)$, the field strength of which vanishes, and vice versa. [The extra condition, used in Sect. 4, that the field $\mathscr{A}$ is representable in the form (4.3) can be neglected here, because it does not constrain essentially the fields on $P$; namely, this condition holds for generic $\mathscr{A}$, as we already mentioned.] Using the vanishing of the field strength of $\left(\mathscr{A}_{\alpha}, \mathscr{A}_{\dot{\alpha}}, \mathscr{A}\right)$ we conclude that this field can be always transformed to a gauge where $\mathscr{A}_{\alpha}=\mathscr{A}_{\dot{\alpha}}=0$. (Note that this is the case, because our consideration is local with respect to coordinates of Minkowski space; note also that the component $\mathscr{A}$ cannot be in general gauged away simultaneously with $\mathscr{A}_{\alpha}, \mathscr{A}_{\dot{\alpha}}$ since we have required the domains of definition of the functions considered to be global in homogeneous coordinates $p^{1}, p^{2}$.) Thus after the gauge transformation we are led to a field of the form $(0,0, \mathscr{A})$ which still has vanishing strength. The last condition reduces, in the case of the field $(0,0, \mathscr{A})$, merely to

$$
D_{\alpha} \mathscr{A}=D_{\dot{\alpha}} \mathscr{A}=0 \text {. }
$$

That is to say, the field $\mathscr{A}$ must be an isochiral function on $P$.

Our gauge condition, $\mathscr{A}_{\alpha}=\mathscr{A}_{\dot{\alpha}}=0$, restricts the group of gauge transformations (4.2) requiring that the gauge function $g$ must be also an isochiral function.

We have thus constructed a correspondence between the gauge equivalence classes of gauge fields on $M$ satisfying the kinematical constraints (2.1) and gauge equivalence classes of homogeneous isochiral fields of degree 2 on $P$. (Gauge transformation for the isochiral field $\mathscr{A}$ is $\mathscr{A} \rightarrow \mathscr{A}^{\prime}=g^{-1}(D+\mathscr{A}) g$ with $g$ being an isochiral function on $P$.) As we have seen, Eq. (5.1) on $P$ can be easily solved using the space $\mathscr{P}$. (This equation and its solution are perfectly analogous to what is usually done with $N=1$ chiral superfields.) This means that the correspondence described here gives us unconstrained superfields for $N=2$ super Yang-Mills theory. To conclude, the Lagrangian for this theory must be described. Unfortunately, there is no simple way known to write down a Lagrangian directly in terms of the unconstrained superfield $\mathscr{A}$. On the other hand, an expression for the Lagrangian in terms of gauge fields $\mathscr{A}_{\alpha i}, \mathscr{A}_{\dot{\alpha}}{ }^{i}$ constrained by (2.1) is well-known [8]. Hence, one has to express constrained superfields, $\mathscr{A}_{\alpha i}, \mathscr{A}_{\dot{\alpha}}^{i}$, in terms of the unconstrained one, $\mathscr{A}$, and substitute into the Lagrangian of [8]. [As one can see from the consideration of the present section, this procedure involves solving Eq. (4.3); if a solution $g$ to (4.3) is known, then the fields $\mathscr{A}_{\alpha i}, \mathscr{A}_{\dot{\alpha}}{ }^{i}$ can be restored from $\left.p^{i} \mathscr{A}_{\alpha i}=g^{-1} D_{\alpha} g, p_{i} \mathscr{A}_{\dot{\alpha}}^{i}=g^{-1} D_{\dot{\alpha}} g.\right]$ The resulting expression for the Lagrangian [1] looks non-local with respect to the axuiliary variables $p^{1}, p^{2}$.

\section{Equations of Motion of the $N=2$ Hypermultiplet}

In this section we consider the so-called hypermultiplet [10], which is an $N=2$ supermultiplet consisting of one isodoublet scalar and two isosinglet (Weyl) spinors. Until recently only an on-shell superfield was known for the hypermultiplet. This is the isodoublet scalar superfield $\varphi_{i}(x, \theta, \bar{\theta})$ satisfying the following constraints.

$$
D_{\alpha i} \varphi_{j}+D_{\alpha j} \varphi_{i}=0, \quad \bar{D}_{\dot{\alpha}}{ }^{i} \varphi_{j}-\frac{1}{2} \delta_{j}^{i} \bar{D}_{\dot{\alpha}}{ }^{k} \varphi_{k}=0
$$


Equations (6.1) imply free massless equations of motion for the component fields. Here we shall first convert the equations of motion (6.1) into equivalent equations [4] on the isotwistor space $P$ (see Sect. 3) and then, in Sect. 7, we shall show following [1] how to separate the resulting equations on $P$ into kinematical constraints and Lagrangian equations of motion. It is important to point out that such unconstrained superspace formulation of the hypermultiplet allows one to introduce also interaction terms into the Lagrangian [1]. In a forthcoming paper we shall describe an off-shell formulation that can be constructed on the superspace $P$ for an arbitrary $N=2$ supersymmetric hyper-Kähler sigma model.

Let us recast Eqs. (6.1) into a different form. If we consider the superfield $\varphi=p^{i} \varphi_{i}$, then the constraints (6.1) on $\varphi_{i}$ imply

$$
D_{\alpha} \varphi=0, \quad D_{\dot{\alpha}} \varphi=0,
$$

where the operators $D_{\alpha}$ and $D_{\dot{\alpha}}$ (depending on $p^{i}$ ) are the same as in Sect. 2. Equations (6.2) satisfied by $\varphi=p^{i} \varphi_{i}$ for all $p^{i}$ are obviously equivalent to (6.1). The superfield $\varphi=\varphi(x, \theta, \bar{\theta}, p, \bar{p})$ can be considered as a function homogeneous of degree one on the superspace $P$. The condition of linearity in the variables $p^{i}$ can be interpreted as the requirement that $\varphi$ is holomorphic with respect to these variables, that is

$$
D \varphi=0
$$

(cf. Sect. 3; as we have already said, a holomorphic function homogeneous of degree one with respect to $p^{i}$ is always linear). Thus we reach the conclusion that the equations of motion (6.1) of the hypermultiplet are equivalent to the system of Eqs. (6.2), (6.3) on the homogeneous field of degree one on $P^{8}$.

\section{Off-Shell Formulation of the Hypermultiplet}

Now it is natural to consider Eqs. (6.2) as kinematical constraints, while the remaining Eq. (6.3) as a true equation of motion to be derived from an action. This was done in [1]. The relevant expression for the Lagrangian is $\tilde{\varphi} D \varphi$, where

$$
\tilde{\varphi}(\hat{p})=[\varphi(\varrho(\hat{p}))]^{*} \text {. }
$$

(See the definitions at the end of Sect. 4.) The two points must be noted in connection with such a candidate Lagrangian. Firstly, since $\varphi$ satisfies kinematical constraints (6.2) (that is to say, it is an isochiral function in the terminology of Sect. 3), so does the conjugated field $\tilde{\varphi}$ and, hence, the Lagrangian density $\tilde{\varphi} D \varphi$. Secondly, the full degree of homogeneity of $\tilde{\varphi} D \varphi$ is +4 . The former remark means that the action involving the Lagrangian $\tilde{\varphi} D \varphi$ must be an integral not over the whole (6|8)-dimensional superspace measure on $P$, but over some (6|4)dimensional measure which implies integration only over the half of fermionic coordinates. The second remark above means that $\tilde{\varphi} D \varphi$ must be integrated with a measure that has the degree of homogeneity equal to minus four to compensate for

${ }^{8}$ One can show that such fields on $P$ correspond to fields on the space $\mathscr{P}$ (see Sect. 3 ) that are holomorphic with respect to all the variables in $\mathscr{P}[4,14]$ 
the degree of $\tilde{\varphi} D \varphi$ (since the integral of a function homogeneous of degree zero only can be defined unambigously when one deals with integration over $\mathbb{C} P^{1}$ in $P=M \times \mathbb{C P}^{1}$.

Now, in coordinates $y^{a}, \Theta^{\alpha}, \Theta^{\dot{\alpha}}, \widetilde{\Theta}^{\alpha}, \widetilde{\Theta}^{\alpha}, p^{i}, \bar{p}_{i}$ the constraints (6.2) mean merely that $\varphi$ does not depend on $\widetilde{\Theta}^{\alpha}$ and $\widetilde{\Theta}^{\alpha}$ (see the discussion in Sect. 3). Consequently, we are led naturally to the fermionic measure $d^{4} \Theta$ which has the correct degree ${ }^{9}$ and does not include $d \widetilde{\Theta}^{\alpha}, d \widetilde{\Theta}^{\dot{\alpha}}$. Thus we arrive at the following expression for the action for the isochiral field $\varphi$,

$$
S=\int d^{4} y^{(0)} d^{2} q^{(0)} d^{4} \Theta^{(-4)} \tilde{\varphi}^{(+1)} D^{(+2)} \varphi^{(+1)}+\text { c.c. },
$$

where the superscripts in brackets indicate the degrees of homogeneity of the entries. The symbol $d^{2} q$ in (7.1) stands for integration with respect to auxiliary variables over the sphere $S^{2} \approx \mathbb{C} P^{1}$. Here the usual measure on two-dimensional sphere is understood:

$$
d^{2} q=\frac{p_{i} d p^{i} \bar{p}^{j} d \bar{p}_{j}}{|p|^{4}}=\frac{d z d \bar{z}}{\left(1+|z|^{2}\right)^{2}},
$$

where $z=p^{1} / p^{2}$ is an ordinary (i.e. not homogeneous) complex coordinate which parametrises $\mathbb{C} P^{1}$ with one point, $p^{2}=0$, excluded. As one can check directly, Eqs. (6.3) indeed follow from the action (7.1). (In doing this it is useful to convince oneself that one can integrate by parts with respect to the "derivative" $D$.) Thus the use of isotwistor superspace $P$ allowed one to obtain an off-shell unconstrained formulation for the $N=2$ hypermultiplet as well.

\section{8. $N=3$ Theory}

The $N=3$ super Yang-Mills theory can be dealt with in a manner analogous to what we discussed in the preceding sections in connection with $N=2$ theories. A relevant interpretation of $N=3$ equations of motion in terms of a superspace with auxiliary variables was suggested in [4]. Using this interpretation one is able to find an unconstrained off-shell formulation of $N=3$ theory. This formulation was found first in [2]. (The results of [2] will be recapitulated in the next section, though using a different notation.)

Let us consider, in analogy with the $N=2$ case, the differential operators

$$
D_{\alpha}=p^{i} D_{\alpha i}, \quad D_{\dot{\alpha}}=u_{i} \bar{D}_{\dot{\alpha}}{ }^{i},
$$

where $D_{\alpha i}, \bar{D}_{\dot{\alpha}}{ }^{i}$ are the usual spinorial derivatives in the $N=3$ Minkowski superspace $M$ (so $i=1,2,3$ ), while $p^{i}, u_{i}$ are complex parameters constrained by the relation

$$
p^{i} u_{i}=0 \text {. }
$$

The vectors (8.1) span a (0|4)-dimensional isosubspace at each point of $M$. It was pointed out in [4] that the equations of motion of $N=3$ gauge fields $\left(\mathscr{A}_{\alpha i}, \mathscr{A}_{\dot{\alpha}}^{i}\right)$ in $M$

${ }^{9}$ The degree of $\Theta^{\alpha}$ and $\Theta^{\dot{\alpha}}$ is plus one, while integration with fermionic measure $d^{4} \Theta$ means differentiation with respect to these coordinates. Hence the degree of $d^{4} \Theta$ is minus four, as required 
are equivalent to the requirement that their field strengths vanish on each isosubspace. That is to say, if we consider the operators

$$
\nabla_{\alpha}=p^{i} \nabla_{\alpha i}, \quad \nabla_{\dot{\alpha}}=u_{i} \nabla_{\dot{\alpha}}^{i},
$$

constructed from the gauge covariant derivatives $\nabla_{\alpha i}=D_{\alpha i}+\mathscr{A}_{\alpha i}, \nabla_{\dot{\alpha}}^{i}=\bar{D}_{\dot{\alpha}}{ }^{i}+\mathscr{A}_{\dot{\alpha}}^{i}$, then the constraints (2.1) on $\nabla_{\alpha i}$, $\nabla_{\dot{\alpha}}^{i}$ (which, for $N=3$, are just the equations of motion) are equivalent to

$$
\left\{\nabla_{\alpha}, \nabla_{\beta}\right\}=\left\{\nabla_{\dot{\alpha}}, \nabla_{\beta}\right\}=\left\{\nabla_{\alpha}, \nabla_{\beta}\right\}=0
$$

satisfies for arbitrary values of $p^{i}, u_{i}$ subjected to (8.2).

This suggests, just as in the $N=2$ case, that one is to pass to the space of all isosubspaces in all points of $M$, i.e. to the isotwistor space $P$. Then the operators (8.3) will define a (partial) gauge field on $P$. For $N=3$ we have $P=M \times Q$, where $Q$ is a compact complex three-dimensional manifold, defined as quadric (8.2) in $\mathbb{C} P^{2}$ $\times \mathbb{C} P^{2}$, in terms of homogeneous coordinates $\left(p^{i}\right)$ and $\left(u_{i}\right)$. The dimension of $P$ equals, thus, 10|12. The use of isotwistor space allows us to obtain a correspondence between gauge fields in $M$ satisfying the constraints (2.1) and certain partial gauge fields in $P$ which have zero field strength.

Before proceeding further it will be useful to consider certain convenient coordinate choices. First of all, in $P=M \times Q$, we have coordinates $x^{a}, \theta^{\alpha i}, \bar{\theta}^{\dot{\alpha}}{ }_{i}, p^{i}, \bar{p}_{i}$, $u_{i}, \bar{u}^{i}$, where $\left(p^{i}\right)$ and $\left(u_{i}\right)$ are homogeneous coordinates constrained by $p^{i} u_{i}=0$, while $\bar{p}_{i}=\left(p^{i}\right)^{*}, \bar{u}^{i}=\left(u_{i}\right)^{*}$. It will be useful to introduce also coordinates for which the operators $D_{\alpha}, D_{\dot{\alpha}}$ given by (8.1) become partial derivatives with respect to some variables. For this purpose, it is instructive to consider the manifold of isoplanes. An isoplane is defined as a (0|4)-dimensional complex submanifold in the complexified Minkowski superspace $M^{c}$ generated by the vector fields (8.1). Such a submanifold can be given by the following parametric equations:

$$
\begin{aligned}
& x^{a}=y^{a}+i \varepsilon^{\alpha} p^{i} \sigma_{\alpha \dot{\beta}}^{a} \eta^{\dot{\beta}}{ }_{i}-i \eta^{\alpha i} \sigma_{\alpha \dot{\beta}}^{a} \varepsilon^{\dot{\beta}} u_{i}, \\
& \theta^{\alpha i}=\eta^{\alpha i}+\varepsilon^{\alpha} p^{i}, \quad \bar{\theta}^{\dot{\alpha}}{ }_{i}=\eta^{\dot{\alpha}}{ }_{i}+\varepsilon^{\dot{\alpha}} u_{i} .
\end{aligned}
$$

Here $y^{a}, \eta^{\alpha i}, \eta^{\dot{\alpha}}, p^{i}, u_{i}$ are constants, while the independent complex (fermionic) variables $\varepsilon^{\alpha}, \varepsilon^{\dot{\alpha}}$ parametrise this isoplane. The isoplane (8.5) can be fixed conveniently also by the following equations:

$$
\begin{gathered}
x^{a}=y^{a}+i \tilde{\Theta}^{\alpha} \sigma_{\alpha \dot{\beta}}^{a} \Theta^{\dot{\beta}}-i \Theta^{\alpha} \sigma_{\alpha \dot{\beta}}^{a} \tilde{\Theta}^{\dot{\beta}} ; \\
u_{i} \theta^{\alpha i}=\Theta^{\alpha}, \quad(0,1) ; \\
|p|^{-2} \bar{p}_{i} \theta^{\alpha i}=\widetilde{\Theta}^{\alpha}, \quad(-1,0) ; \\
p^{i} \bar{\theta}^{\dot{\alpha}}{ }_{i}=\Theta^{\dot{\alpha}}, \quad(1,0) ; \\
|u|^{-2} \bar{u}^{i} \bar{\theta}^{\dot{\alpha}}{ }_{i}=\widetilde{\Theta}^{\dot{\alpha}}, \quad(0,-1) ; \\
|u|^{-2} \varepsilon_{i j k} p^{i} \bar{u}^{j} \theta^{\alpha k}=\Phi^{\alpha}, \quad(1,-1) ; \\
|p|^{-2} \varepsilon^{i j k} \bar{p}_{i} u_{j} \bar{\theta}^{\dot{\alpha}}{ }_{k}=\Phi^{\dot{\alpha}}, \quad(-1,1) ;
\end{gathered}
$$

where $|p|^{2} \equiv p^{i} \bar{p}_{i} \neq 0$ and $|u|^{2} \equiv u_{i} \bar{u}^{i} \neq 0$, and now $y^{a}, \Theta^{\alpha}, \Theta^{\alpha}, \Phi^{\alpha}, \Phi^{\dot{\alpha}}, p^{i}, u_{i}$ are the constants that define an isoplane uniquely, while $\widetilde{\Theta}^{\alpha}, \widetilde{\Theta}^{\dot{\alpha}}$ are the parameters on it. Thus $y^{a}, \Theta^{\alpha}, \Theta^{\dot{\alpha}}, \Phi^{\alpha}, \Phi^{\dot{\alpha}}, p^{i}, u_{i}$ build up a coordinate system in the manifold $\mathscr{P}$ of all 
isoplanes in $M^{c}$. Note, that these coordinates in $\mathscr{P}$ have certain degrees of homogeneity ${ }^{10}$ indicated in brackets in Eqs. (8.6). The space $P$ can be considered as a submanifold in $\mathscr{P}$. Geometrically, the embedding $P \subset \mathscr{P}$ can be defined as follows. As one can see from (8.6), every isoplane in $M^{c}$ intersects the real submanifold $M$ of $M^{c}$ in at most one point. Hence, such an isoplane is defined uniquely by a tangent isosubspace (at some point) in $M$. This gives us just an embedding of $P$ into $\mathscr{P}$. The resulting submanifold $P$ in $\mathscr{P}$ can be defined also by means of the following equations:

$$
\begin{gathered}
y^{a}-\left(y^{a}\right)^{*}=2 i \Theta^{\alpha} \sigma_{\alpha \dot{\beta}}^{a} \tilde{\Theta}^{\dot{\beta}}-2 i \tilde{\Theta}^{\alpha} \sigma_{\alpha \dot{\beta}}^{a} \Theta^{\dot{\beta}}, \\
\Phi^{\dot{\alpha}}=|u|^{2}|p|^{-2}\left(\Phi^{\alpha}\right)^{*},
\end{gathered}
$$

where

$$
\tilde{\Theta}^{\dot{\alpha}}=|u|^{-2}\left(\Theta^{\alpha}\right)^{*}, \quad \Theta^{\dot{\alpha}}=|p|^{2}\left(\widetilde{\Theta}^{\alpha}\right)^{*} .
$$

In terms of coordinates $y, \Theta, \Phi, \widetilde{\Theta}, p, \bar{p}, u, \bar{u}$, the operators (8.1) become

$$
D_{\alpha}=\partial / \partial \tilde{\Theta}^{\alpha}, \quad D_{\dot{\alpha}}=\partial / \partial \tilde{\Theta}^{\dot{\alpha}} .
$$

Thus, if we consider an $N=3$ isochiral function on $P$, that is a function $f$ which satisfies

$$
D_{\alpha} f=D_{\dot{\alpha}} f=0,
$$

then these constraints will mean that $f$ does not depend on $\widetilde{\Theta}$. (In other words, an isochiral function on $P$ can be continued to a function on $\mathscr{P}$ holomorphic with respect to the variables $y, \Theta, \Phi$.)

Let us consider now the three-dimensional compact complex manifold $Q$ introduced above. As in the case of the manifold $\mathbb{C} P^{1}$ (dealt with in Sect. 3), we may consider various homogeneous functions on $Q$. In the present case we have also the following useful proposition. Every homogeneous holomorphic function of degree $(m, n)$ on $Q$ is a homogeneous polynomial of $m^{\text {th }}$ order in $p^{i}$ and of $n^{\text {th }}$ order in $u_{i}$. Let us introduce the differential operators

$$
\begin{aligned}
& X_{1}=|p|^{2}|u|^{-2} \varepsilon_{i j k} p^{i} \bar{u}^{j} \frac{\partial}{\partial \bar{p}_{k}}, \quad(2,-1) \\
& X_{2}=|p|^{-2}|u|^{2} \varepsilon^{i j k} \bar{p}_{i} u_{j} \frac{\partial}{\partial \bar{u}^{k}}, \quad(-1,2) \\
& X_{3}=|p|^{2} u_{i} \frac{\partial}{\partial \bar{p}_{i}}-|u|^{2} p^{i} \frac{\partial}{\partial \bar{u}^{i}},
\end{aligned}
$$

which obey the following algebra:

$$
\left[X_{1}, X_{2}\right]=X_{3}, \quad\left[X_{1}, X_{3}\right]=\left[X_{2}, X_{3}\right]=0 \text {. }
$$

In (8.9) the degrees of homogeneity were indicated in brackets. If $z^{s}, s=1,2,3$, are some arbitrary complex coordinates defined locally on $Q$, then one has for a

\footnotetext{
${ }^{10}$ In the present case the degree of homogeneity must be characterized by two integers $(m, n)$ corresponding to two subsets of homogeneous coordinates, respectively $\left(p^{i}\right)$ and $\left(u_{i}\right)$. The rest is quite analogous to dealing with homogeneous functions on $\mathbb{C} P^{1}$, as explained in Sect. 3
} 
(homogeneous of any degree) function $f$ on $Q$

$$
\bar{\partial} f \equiv \frac{\partial f}{\partial \bar{z}^{s}} d \bar{z}^{s}=X_{s} f \cdot \omega^{s}
$$

where $\left(\omega^{s}\right)$ is the following set of three homogeneous 1 -forms on $Q$,

$$
\begin{gathered}
\omega^{1}=|p|^{-4} \varepsilon^{i j k} \bar{p}_{i} u_{j} d \bar{p}_{k}, \\
\omega^{2}=|u|^{-4} \varepsilon_{i j k} p^{i} \bar{u}^{j} d \bar{u}^{k}, \\
\omega^{3}=|u|^{-2}|p|^{-2} \bar{u}^{j} d \bar{p}_{j} \equiv-|u|^{-2}|p|^{-2} \bar{p}_{j} d \bar{u}^{j} .
\end{gathered}
$$

Thus the requirement that a function $f$ be holomorphic on $Q$ is equivalent to the equations

$$
X_{s} f=0, \quad s=1,2,3 .
$$

We shall use also the operators $X_{s}$ on the space $P=M \times Q$. [In terms of coordinates $x^{a}, \theta^{\alpha i}, \bar{\theta}^{\dot{\alpha}}{ }_{i}, p^{i}, \bar{p}_{i}, u_{i}, \bar{u}^{i}$ these operators are defined on $P$ by Eqs. (8.9).]

We are ready now to reformulate the $N=3$ equations of motion (2.1) in terms of the isotwistor space. This reformulation uses the partial gauge field $\left(\mathscr{A}_{\alpha}, \mathscr{A}_{\dot{\alpha}}, \mathscr{A}_{\mathrm{s}}\right)$ that gauges the differential operators $(8.1),(8.9)$ on $P$ giving

$$
\nabla_{\alpha}=D_{\alpha}+\mathscr{A}_{\alpha}, \quad \nabla_{\dot{\alpha}}=D_{\dot{\alpha}}+\mathscr{A}_{\dot{\alpha}}, \quad \nabla_{X_{s}}=X_{s}+\mathscr{A}_{s} .
$$

As for $N=2$, we obtain a one-to-one correspondence [4] between (the gauge equivalence classes of) gauge fields $\left(\mathscr{A}_{\alpha i}, \mathscr{A}_{\dot{\alpha}}{ }^{i}\right.$ ) satisfying Eqs. (2.1) on $M$ and (the equivalence classes of) partial gauge fields $\left(\mathscr{A}_{\alpha}, \mathscr{A}_{\dot{\alpha}}, \mathscr{A}_{s}\right)$ on $P=M \times Q$, which have vanishing field strengths ${ }^{11}$. The latter condition means that the operators (8.13) satisfy the same (anti)commutation relations as do the "flat" operators (8.1), (8.9). That is to say, the relations (8.4) must hold along with

$$
\begin{gathered}
{\left[\nabla_{X_{s}}, \nabla_{\alpha}\right]=\left[\nabla_{X_{s}}, \nabla_{\dot{\alpha}}\right]=0,} \\
{\left[\nabla_{X_{1}}, \nabla_{X_{2}}\right]=\nabla_{X_{3}}, \quad\left[\nabla_{X_{1}}, \nabla_{X_{3}}\right]=\left[\nabla_{X_{2}}, \nabla_{X_{3}}\right]=0 .}
\end{gathered}
$$

There is a convenient gauge choice; namely, $\mathscr{A}_{\alpha}=\mathscr{A}_{\dot{\alpha}}=0$. Then Eqs. (8.14) become

$$
D_{\alpha} \mathscr{A}_{s}=D_{\dot{\alpha}} \mathscr{A}_{s}=0 \text {, }
$$

which means merely that, in this gauge, $\mathscr{A}_{s}$ are isochiral functions on $P$. [Note also, that $\mathscr{A}_{s}$ have certain degrees of homogeneity, namely, those of corresponding $X_{s}$, cf. (8.9).]

It remains to specify the reality conditions on the partial gauge fields on $P$ that must be imposed if the gauge group $G$ is real. If, for example, $G=\mathrm{SU}(n)$, then the gauge fields on Minkowski superspace $M$ satisfy $\mathscr{A}_{\dot{a}}{ }^{i}=-\left(\mathscr{A}_{\alpha i}\right)^{+}$. The corresponding reality condition on the isotwistor space $P$ turns out to be as follows:

$$
\mathscr{A}_{\dot{\alpha}}(\hat{p})=-\left[\mathscr{A}_{\alpha}(\varrho(\hat{p}))\right]^{+},
$$

${ }^{11}$ In fact, we should have imposed an additional condition that $\mathscr{A}_{a}=g^{-1} X_{s} g$ for some gauge function $g$ on $P$. As for $N=2$, this condition can be neglected in perturbation theory. The difference between the $N=3$ and $N=2$ cases beyond perturbation theory will be discussed in Sect. 11 
and for components $\mathscr{A}_{s}, s=1,2,3$,

$$
\mathscr{A}_{1}(\hat{p})=-\left[\mathscr{A}_{2}(\varrho(\hat{p}))\right]^{+}, \quad \mathscr{A}_{3}(\hat{p})=\left[\mathscr{A}_{3}(\varrho(\hat{p}))\right]^{+},
$$

where $\hat{p}$ denotes a point in $P$ with coordinates $\left(x^{a}, \theta^{\alpha i}, \bar{\theta}^{\dot{\alpha}}, p^{i}, \bar{p}_{i}, u_{i}, \bar{u}^{i}\right)$ and the transformation $\varrho$ replaces $p^{i}$ by $\bar{u}^{i}$, that is $\hat{p} \rightarrow \varrho(\hat{p})=\left(x^{a}, \theta^{\alpha i}, \bar{\theta}^{\alpha} i, \bar{u}^{i}, u_{i}, \bar{p}_{i}, p^{i}\right)$.

\section{9. $N=3$ Theory Off-Shell}

In the last section we saw that the $N=3$ equations of motion can be reformulated as Eqs. (8.15), (8.16) on the superfields $\mathscr{A}_{s}$ defined on isotwistor space $P$. In order to obtain an off-shell formulation of this theory it remains, as suggested in [2], to find an action from which Eqs. (8.15) can be derived as the Euler-Lagrange equations. Then the remaining Eqs. (8.16) must be interpreted as kinematical constraints that are easy to solve, as we have already mentioned. Equations (8.15) can be rewritten as

$$
\begin{gathered}
F_{12} \equiv X_{1} \mathscr{A}_{2}-X_{2} \mathscr{A}_{1}+\left[\mathscr{A}_{1}, \mathscr{A}_{2}\right]-\mathscr{A}_{3}=0, \\
F_{13} \equiv X_{1} \mathscr{A}_{3}-X_{3} \mathscr{A}_{1}+\left[\mathscr{A}_{1}, \mathscr{A}_{3}\right]=0, \\
F_{23} \equiv X_{2} \mathscr{A}_{3}-X_{3} \mathscr{A}_{2}+\left[\mathscr{A}_{2}, \mathscr{A}_{3}\right]=0 .
\end{gathered}
$$

Let us look for an action which satisfies

$$
\delta S=\int_{P} d \mu \varepsilon^{s t u} \operatorname{tr} F_{s t} \delta \mathscr{A}_{u}+\text { c.c. },
$$

where $F_{s t}$ are defined in (9.1), and $d \mu$ is an appropriate measure on the superspace $P$. Note, that since $\mathscr{A}_{s}$ has the same degree of bomogeneity as that of $X_{s}$, the degree of the integrand in (9.2) equals the sum of degrees of $X_{1}, X_{2}$ and $X_{3}$ [cf. (8.9)]. Thus the degree of $\varepsilon^{s t u} F_{s t} \delta \mathscr{A}_{u}$ equals $(2,-1)+(-1,2)+(1,1)=(2,2)$. This implies that the integration measure $d \mu$ in $(9.2)$ must be of degree $(-2,-2)$. [Otherwise, the integral (9.2) over $P=M \times Q$ could not be defined unambiguously.] Moreover, since the superfields $\mathscr{A}_{s}$ (and, hence, $F_{s t}$ ) are isochiral, the measure $d \mu$ cannot include $d \widetilde{\Theta}$, as one can see from Eqs. (8.16) using (8.8). Thus we are forced to choose $^{12}$

$$
d^{10 \mid 8} \mu=d^{4} y d^{6} q d^{4} \Theta d^{4} \Phi,
$$

where we assume the use of coordinates $y, \Theta, \Phi, \tilde{\Theta}, p, \bar{p}, u, \bar{u}$ (defined in Sect. 8). The symbol $d^{6} q$ in (9.3) stands for integration with respect to auxiliary variables over the manifold $Q$ of real dimension six. The measure $d^{6} q$ on $Q$ can be defined either using the embedding $Q \subset \mathbb{C} P^{2} \times \mathbb{C} P^{2}$, or, equivalently, as the unique (up to a constant factor) $\mathrm{SU}(3)$ invariant measure on $Q$. (Note, that $Q$ is isomorphic to $\mathrm{SU}(3) /[\mathrm{U}(1) \times \mathrm{U}(1)])$. Let us introduce on $Q$ local complex coordinates $z^{s}$, $s=1,2,3$, by means of relations $z^{1}=p^{1} / p^{3}, z^{2}=p^{2} / p^{3}, z^{3}=u_{1} / u_{2}$. Then

$$
\begin{aligned}
d^{6} q & =-|p|^{4}|u|^{4} \omega^{1} \omega^{2} \omega^{3} \bar{\omega}^{1} \bar{\omega}^{2} \bar{\omega}^{3} \\
& =\frac{d^{3} z d^{3} \bar{z}}{\left(1+\left|z^{1}\right|^{2}+\left|z^{2}\right|^{2}\right)^{4}\left(1+\left|z^{3}\right|^{2}+\left|z^{2}-z^{1} z^{3}\right|^{2}\right)^{2}},
\end{aligned}
$$

\footnotetext{
${ }^{12}$ Note that for any fermionic coordinate, say, $\Theta$, the degree of homogeneity of $d \Theta$ is minus the degree of $\Theta$
} 
where the 1 -forms $\omega^{s}$ are defined in (8.11). Now the action obeying (9.2) can be found and turns out to be as follows:

$$
\begin{aligned}
S= & \int_{P} d \mu \operatorname{tr}\left\{-\frac{1}{2} \mathscr{A}_{3}^{2}+\mathscr{A}_{3}\left(X_{1} \mathscr{A}_{2}-X_{2} \mathscr{A}_{1}\right)\right. \\
& \left.-\mathscr{A}_{1} X_{3} \mathscr{A}_{2}+\mathscr{A}_{3}\left[\mathscr{A}_{1}, \mathscr{A}_{2}\right]\right\}+ \text { c.c. }
\end{aligned}
$$

Remember, that $\mathscr{A}_{s}$ in (9.4) are the components of the partial gauge field corresponding to differential operators $X_{s}$; see (8.9). Using local coordinates $z^{s}$ one can rewrite the action (9.4) in terms of components of the partial gauge field corresponding to differential operators $\bar{\partial}_{s} \equiv \partial / \partial \bar{z}^{s}$ as well. Then the action acquires a Chern-Simons-like form of Eq. (1.3).

The gauge transformations of superfields $\mathscr{A}_{s}$ which are compatible with kinematical constraints (8.16) and leave the equations of motion (9.1) invariant are obviously as follows:

$$
\mathscr{A}_{s} \rightarrow g^{-1} \mathscr{A}_{s} g+g^{-1} X_{s} g,
$$

where the gauge function $g$ on the space $P$ is isochiral, i.e. $D_{\alpha} g=D_{\dot{\alpha}} g=0$, and satisfies a reality condition corresponding to (8.17):

$$
[g(\hat{p})]^{-1}=[g(\varrho(\hat{p}))]^{+} .
$$

The action (9.4) is invariant under gauge transformations (9.5), although the invariance is non-manifest: the integrand in (9.4) transforms by a total derivative under (9.5). This property of the action (9.4) is analogous to that of the ChernSimons term [9]. It can be shown, however, that in contrast to the Chern-Simons term the action (9.4) is invariant even under "large" non-Abelian gauge transformations ${ }^{13}$. Thus there is no topological quantisation of the coefficient in front of the action (9.4) (as one probably could expect from the analogy with the ChernSimons term, which yields the topologically quantised mass to three-dimensional non-abelian gauge theories [9]).

\section{Gauge Superfields as CR-Bundles}

As it was already pointed out in Sect. 1, the way of constructing various superfield theories we dealt with in this paper is based on an idea due to Ward. As a matter of fact, the original work of Ward [3] establishes a one-to-one correspondence between self-dual gauge fields and certain holomorphic bundles over a special space with auxiliary dimensions. (In that case the space with auxiliary dimensions, namely, the projective twistor space $\mathbb{C} P^{3}$, emerges, when one adds in a definite way an auxiliary manifold $\mathbb{C} P^{1}$ to each point of the sphere $S^{4}$ ). Other works exploiting Ward's idea in different situations also used the language of holomorphic bundles (e.g., $[6,7]$ ) or CR-bundles ${ }^{14}[4]$. There exist various ways well known in mathematics (e.g. [11]) to give the formal definitions of such bundles. One of these

\footnotetext{
${ }^{13}$ This is due to the fact that the set of homotopy classes of maps of $Q$ into $G$ (the gauge group) is finite

${ }^{14}$ The difference in using holomorphic bundles or CR-bundles is purely technical, as it will be explained below
} 
definitions uses just the geometrical objects that were referred to in preceding sections as partial gauge fields. It is this manner of description of holomorphic bundles which leads directly to unconstrained superfield formulations of $[1,2]$, as we hope we have shown in this paper ${ }^{15}$. The present section is intended to explain the interrelations between various definitions of holomorphic bundles. After that it will be clear, in particular, that the results of [4] expressed there in terms of CRbundles over the isotwistor superspace $P$ imply the formulation of super YangMills constrains in terms of partial gauge fields on $P$.

Let us recall [11], that a complex vector bundle over some manifold $\mathscr{M}$ can be defined by gluing together (with the help of gauge transformations) the direct products $U_{i} \times \mathbb{C}^{n}$. Here $U_{i}$ are open subsets covering $\mathscr{M}$. If $\mathscr{M}$ is a complex manifold, it is natural to consider the case when the transition functions (i.e. gauge transformations used for gluing) are holomorphic. In that case the vector bundle under consideration is called holomorphic. To get another useful definition, let us consider sections of a vector bundle (with $\mathbb{C}^{n}$ as a fibre). Locally, a section $\psi$ can always be represented by an $n$-component complex field $\psi^{\alpha}(x), \alpha=1, \ldots, n$, and there is still the possibility to make a gauge transformation $\psi^{\alpha}(x) \rightarrow g_{\beta}^{\alpha}(x) \psi^{\beta}(x)$ with $g_{\beta}^{\alpha}(x)$ being a function with values in $\operatorname{GL}(n, \mathbb{C})$ (or in some subgroup of it). If $\mathscr{M}$ is a complex manifold, let $z^{s}, s=1, \ldots, m$, be some local complex coordinates in it. A holomorphic function $f(z)$ on (a domain in) $\mathscr{M}$ satisfies by definition the equation $\bar{\partial}_{s} f \equiv \partial f / \partial \bar{z}^{s}=0$. Suppose, one wishes to define the notion of a holomorphic section. For this definition to be gauge invariant, it has to be of the following form:

$$
\nabla_{s} \psi \equiv\left(\bar{\partial}_{s}+\mathscr{A}_{s}\right) \psi=0
$$

where $\psi$ is a section, and in every gauge $\psi$ is represented by some fields $\psi^{\alpha}$, while $\mathscr{A}_{s}$ are represented by $n \times n$ matrix valued fields $\mathscr{A}_{s \beta}^{\alpha}$. The transformation law of $\mathscr{A}_{s}$ must be obviously

$$
\mathscr{A}_{s} \rightarrow g \mathscr{A}_{s} g^{-1}+g \bar{\partial}_{s} g^{-1} .
$$

That is to say, the definition of holomorphic sections requires us to introduce a partial gauge field corresponding to the differential operators $\bar{\partial}_{s}$. We observe immediately, that the definition of holomorphic sections (10.1) will provide us with a reasonable set of such sections if

$$
\left[\nabla_{s}, \nabla_{r}\right]=0
$$

for any $s, r=1, \ldots, m$. Indeed, Eq. (10.2) is the integrability condition for the system of partial differential equations (10.1). If it were not imposed, there might be, even locally, too little (possibly no) holomorphic sections.

The discussion above justifies the following definition. A holomorphic vector bundle over complex manifold $\mathscr{M}$ is a vector bundle (with $\mathbb{C}^{n}$ as a fibre) provided with a partial gauge field which enters covariant derivatives $\nabla_{s}=\bar{\partial}_{s}+\mathscr{A}_{s}$ satisfying $(10.2)^{16}$. (This is equivalent to the definition given above in terms of holomorphic

\footnotetext{
${ }^{15}$ Such treatment of holomorphic bundles was successfully used also previously in twistorial constructions; see [12]

${ }^{16}$ If the vector bundle under consideration is topologically non-trivial, one has to consider, as usual, a number of local charts related by gauge transformations on the intersections. Being topologically trivial, a bundle can be still non-trivial holomorphically (see below). In what follows we shall deal mainly with holomorphic bundles which are topologically trivial
} 
transition functions, as we shall see shortly.) A holomorphic bundle over $\mathscr{M}$ is said to be trivial if the corresponding partial gauge field is a pure gauge; that is

$$
\mathscr{A}_{s}=g^{-1} \bar{\partial}_{s} g \text {, }
$$

where $g$ is an $n \times n$ matrix function defined globally on the manifold $\mathscr{M}$. In fact, one can see that Eq. (10.3) can always be satisfied locally, for the field $\mathscr{A}_{s}$ is assumed to obey (10.2), the integrability condition for (10.3).

If the field $\mathscr{A}_{s}$ is not a pure gauge globally though, one can still use a number of open subsets $U_{i}$ covering $\mathscr{M}$, such that on each of them a solution $g_{i}$ to Eq. (10.3) does exist. Then the matrix valued functions $\varphi_{i j}=g_{i} g_{j}^{-1}$ defined on the intersections $U_{i} \cap U_{j}$ satisfy obviously $\bar{\partial}_{s} \varphi_{i j}=0$ and $\varphi_{i j} \varphi_{j k}=\varphi_{i k}$. In fact, a set of functions (called transition functions) satisfying these conditions defines a holomorphic bundle uniquely. This yields an alternative formal definition of the notion of a holomorphic bundle formulated in terms of holomorphic transition functions ${ }^{17}$.

We observe that locally one can always choose a gauge in which the partial gauge field defining a holomorphic bundle vanishes; such different gauge choices are related by holomorphic gauge transformations. A holomorphic section $\psi$ is represented then in such a gauge by an $n$-component holomorphic field $\psi^{\alpha}(z)$ (i.e. $\left.\bar{\partial}_{s} \psi^{\alpha}=0\right)$.

The notion of a holomorphic function has a natural generalization. Let us consider first a real surface $\Omega$ in a complex manifold $\mathscr{N}$. The functions on $\Omega$ that can be analytically continued to a neighbourhood of $\Omega$ will be called CR-functions. Every operator of the form $X^{i}(u) \partial / \partial \bar{z}^{i}$ gives zero on any holomorphic function on $\mathscr{N}$. (Here $u$ denotes a point in $\mathscr{N}$ with complex coordinates $z^{i}$.) If, for $u$ in $\Omega$, the vector with components $X^{i}(u)$ is tangent to $\Omega$, then the action of the operator $\hat{X}=X^{i}(u) \partial / \partial \bar{z}^{i}$ is defined also on the space of functions on $\Omega$. This allows us to give an internal definition of CR-functions, using the fact that these functions satisfy $\hat{X} f=0$ for any operator $\hat{X}$ of the type considered. Namely, let us consider a manifold $\mathscr{M}$ on which a set of complex vector fiels $Y_{s}, s=1, \ldots, p$, is fixed. (Here we assume that these vectors span at each point a $\dot{p}$-dimensional complex subspace in the complexified tangent space, and that the conjugated vector $Y_{s}$, for any $s$, cannot be expressed as a complex linear combination of $Y_{r}, r=1, \ldots, p$.) In such a case one says that the manifold $\mathscr{M}$ is provided with a CR-structure (i.e. Cauchy-Riemann structure). A CR-structure is called integrable if

$$
\left[Y_{s}, Y_{r}\right]=c_{s r}^{t} Y_{t}
$$

\footnotetext{
${ }^{17}$ This definition is parallel to the definition of a topological bundle by means of transition functions which are required to be continuous. For a given topological (respectively holomorphic) bundle, one can alter the transition functions by making arbitrary continuous (respectively holomorphic) gauge transformations on any open subset $U_{i}$. If by means of these transformations all the transition functions can be simultaneously set to unity (i.e. constant unit matrix), the bundle under consideration is trivial. It may happen that the holomorphic transition functions of a given holomorphic bundle can be gauged away by means of continuous transformations, but cannot by means of holomorphic ones. This is just the case we have already mentioned, when a bundle is trivial topologically, but non-trivial holomorphically
} 
for some function $c_{s r}^{t}$ on $\mathscr{M}$. In this case $\mathscr{M}$ is called a CR-manifold ${ }^{18}$. A function $f$ on $\mathscr{M}$ is called a CR-function if $Y_{s} f=0$ for any $s=1, \ldots, p$. Note, that the condition (10.4) is intended to ensure that on CR-manifolds there exist (locally) sufficiently many CR-functions. It is worth noticing that a generic real surface $\Omega$ in a complex space can be considered as a CR-manifold. (One must take a maximal set of linearly independent vectors $\hat{X}$ on $\Omega$ of the form considered above as vectors $Y_{s}$ defining CR-structure.) In fact, any CR-manifold can be represented in such a way, provided the vector fields $Y_{s}$ are real-analytic.

On a CR-manifold it is natural to consider CR-bundles, a proper generalization of holomorphic bundles over the complex manifold. Namely, one can define CR-bundles as such that the transition functions are CR-functions. The definition of holomorphic bundles in terms of partial gauge fields we have discussed above can also be generalised to the case of CR-bundles. A vector CR-bundle over a CRmanifold $\mathscr{M}$ is a complex vector bundle for which a partial gauge field $\mathscr{A}_{s}$, $s=1, \ldots, p$, corresponding to gauge covariant operators $\nabla_{s}=Y_{s}+\mathscr{A}_{s}$ is fixed, provided

$$
\left[\nabla_{s}, \nabla_{r}\right]=c_{s r}^{t} \nabla_{t}
$$

We are able to give also the definition of CR-sections of a CR-bundle. These are the sections, $\psi$, which satisfy

$$
\nabla_{s} \psi \equiv\left(Y_{s}+\mathscr{A}_{s}\right) \psi=0 .
$$

This definition is reasonable because of the integrability condition (10.5). Other properties of CR-bundles as well as the proof of equivalence of two ways of defining them are straightforward analogs of those for holomorphic bundles. In particular, one can consider the gauges in which $\mathscr{A}_{s}=0$ (locally) and the remaining gauge freedom is given by CR-functions.

Now it is easy to see that the whole of constructions used to convert the Minkowski space superfields into superfields on isotwistor space can be translated in terms of CR-bundles. Let us explain, for simplicity, the case of $N=2$ super YangMills theory. First of all, we observe that for $N=2$ the isotwistor superspace $P=M$ $\times \mathbb{C} P^{1}$ (see Sect. 3) has the following CR-structure. This CR-structure is defined by complex vector fields $D_{\alpha}, D_{\beta}, D$ on $P$ (the dimension of the corresponding complex subspace being, thus, $1 \mid 4)$. Note, that since these vector fields form an algebra closed under the (anti)commutator, the space $P$ is a CR-manifold. The rest is straightforward. Namely, the partial gauge fields $\mathscr{A}_{\alpha}, \mathscr{A}_{\dot{\alpha}}, \mathscr{A}$ on $P$, which substitute the usual superfields on Minkowski superspace $M$ (see, Sect. 4), satisfy the condition of vanishing field strength. That is to say, these partial gauge fields define a CR-bundle ${ }^{19}$ over the CR-manifold $P$. For non-trivial gauge fields the

\footnotetext{
${ }^{18}$ Note that the notion of a CR-structure (respectively integrable CR-structure, respectively CRmanifold) is a direct analog of the notion of an almost complex structure (respectively complex structure, respectively complex manifold). Note, also, that one usually assumes that the two sets of vector fields, say $Y_{s}$ and $Y_{s}^{\prime}$, correspond to the same CR-structure if they generate the same tangent subspace at each point

${ }^{19}$ Since we consider uncompactified Minkowski superspace, the bundles under consideration are topologically trivial
} 
corresponding CR-bundles are non-trivial over $P$, but simultaneously they must be trivial over every submanifold $\mathbb{C} P^{1}$ in $P=M \times \mathbb{C} P^{1}$ corresponding to each fixed point in $M$. The latter condition was expressed in Sect. 4 as the requirement that the component $\mathscr{A}$, which enters $\nabla=D+\mathscr{A}$, is a pure gauge (4.3) globally on $P$.

Let us note, that the resulting interpretation of constrained gauge fields on $M$ in terms of CR-bundles over $P$ also yields a solution of $N=2$ kinematical constraints. Indeed, an arbitrary $\mathrm{CR}$-bunlde over $P=M \times \mathbb{C} P^{1}$ can be defined by means of a single transition function (which must be a CR-function). This is related to the fact that $\mathbb{C} P^{1}$ can be viewed as two copies of one dimensional complex space $\mathbb{C}^{1}$ glued. This transition function can be used as an unconstrained superfield of $N=2$ super Yang-Mills theory.

Finally, it must be noticed that in general one can consider holomorphic (or CR) bundles satisfying some reality conditions. In particular, the condition (4.5) for the partial gauge field defining a CR-bundle on $P$ can be interpreted as a reality condition on this bundle. [For $N=3$, an analogous reality condition was given by Eqs. (8.17).]

Let us mention also a proper geometrical setting of isochiral superfields on $P$ corresponding to the $N=2$ hypermultiplet. Evidently, since an on-shell superfield representing the hypermultiplet satisfies Eqs. (6.2), (6.3), it is just a CR-field on $P$. (An off-shell superfield which satisfies only the isochirality condition (6.2) on $P$, can also be viewed as a CR-field, if one uses a CR-structure on $P$ defined by the vector fields $D_{\alpha}, D_{\dot{\alpha}}$ only.) It remains to point out that since the superfields in this case have a non-zero degree of homogeneity, they correspond not to ordinary CR-functions on $P$, but to CR-sections of a fixed CR-bundle with one-dimensional fibres over $P$.

As we have already pointed out a CR-manifold can always be realized as a surface in certain complex space. Analogously, CR-bundles over this CR-manifold can be described as restrictions to the corresponding surface of holomorphic bundles defined over a neighbourhood of this surface in the ambient complex space. This follows from the fact that CR-functions correspond to holomorphic functions restricted to a real surface. (To be more accurate, all the statements of such a kind in general are valid locally and under the assumption of real analyticity. In case of present interest, it is straightforward to check that the CRstructure, defined, as above, by the vector fields $D_{\alpha}, D_{\dot{\alpha}}, \bar{\partial}_{s}$ on $P($ for $N=2$ and $N=3$ ) is equivalent to the CR-structure arising on the real surface in complex manifold ${ }^{20}$ $\mathscr{P}$. Consequently, all the constructions of the present paper (as well as of [4]) can be reformulated also in terms of holomorphic bundles over $\mathscr{P}$ (see [14] for details).

\section{Discussion of Equivalence Between Minkowski Space and Isotwistor Space}

Let us consider partial gauge fields $\bar{\partial}_{s}+\mathscr{A}_{s}$ of zero field strength on a complex manifold. As we have discussed in Sect. 10, the gauge equivalence classes of such fields are in one-to-one correspondence with holomorphic bundles over $\mathscr{M}$ considered up to holomorphic equivalence. The fields $\mathscr{A}_{s}$ are assumed to be

${ }^{20}$ The supermanifold $\mathscr{P}$ is a complex supermanifold, for it is defined as the manifold of complex submanifolds, isoplanes, in complexified Minkowski superspace (cf. Sects. 3 and 8) 
defined in terms of local coordinates $z^{s}$ on $\mathscr{M}$. If in a domain of $\mathscr{M}$ there are two coordinate systems, corresponding fields must be gauge equivalent. If, moreover, the differential form $\mathscr{A}=\mathscr{A}_{s} d \bar{z}^{s}$ remains the same under the changes of local coordinates, then the corresponding holomorphic bundle is topologically trivial. It is just the case in those constructions involving partial gauge fields on $\mathbb{C} P^{1}$ and $Q$, which we discussed in Sects. 4, 5 and 8, 9. The classification of holomorphic bundles over $\mathbb{C} P^{1}$ is well known [11]. Every one-dimensional bundle over $\mathbb{C} P^{1}$ is holomorphically equivalent to a bundle defined by the transition function $\varphi(z)=z^{n}$ for an integer $n$. [That is to say, this bundle can be built up from two copies of the direct product $\mathbb{C}^{1} \times \mathbb{C}^{1}$ by identifying $(z, u)$ with $\left(z^{-1}, z^{n} u\right)$.] Every $k$-dimensional bundle over $\mathbb{C} P^{1}$ is holomorphically equivalent to a direct sum of one-dimensional bundles. The equivalence class of this bundle is defined by $k$ integers, $\left(n_{1}, \ldots, n_{k}\right)$. A bundle is topologically trivial if $n_{1}+\ldots+n_{k}=0$. It follows that for $k>1$ there exist topologically trivial bundles which are holomorphically non-trivial. This means that a gauge transformation which sets the given field $\mathscr{A}$ of Sect. 4 to zero may not exist for some $\mathscr{A}$. However, the fields, $\mathscr{A}$, on $\mathbb{C} P^{1}$, for which such gauge transformations do exist, can be shown to constitute an open dense subset in the space of all fields.

There is no known classification of holomorphic bundles over the manifold $Q$ that arises in the case of $N=3$ gauge theory (Sect. 8), unless the dimension of fibres equals one. All the one-dimensional topologically trivial bundles over $Q$ are holomorphically trivial. Indeed, discussion of Sect. 10 implies that such onedimensional bundles over any complex manifold $\mathscr{M}$ are in one-to-one correspondence with equivalence classes of 1 -forms $\omega=\omega_{s} d \bar{z}^{s}$ obeying the condition $\bar{\partial} \omega=0$ (here $\omega$ and $\omega+\bar{\partial} \varrho$ are assumed to be equivalent). The set of equivalence classes of such 1 -forms is denoted in mathematics as $H^{0,1}(\mathscr{M})$. It can be derived from some known mathematical facts that $H^{0,1}(Q)=0$. In the case of an arbitrary dimension of fibres, it follows from the theory of deformations of complex bundles [13] that any holomorphic bundle sufficiently close to a holomorphically trivial bundle over $Q$ is itself holomorphically trivial. In other words, if a sufficiently small field $\mathscr{A}_{s}$ on $Q$ has vanishing field strength, then it is gauge equivalent to zero. (One can prove this by means of a perturbation theory.) Thus the set of partial gauge fields corresponding to holomorphically trivial bundles over $Q$ is open in the space of all fields. However, it is not a dense subset in the present case, unlike the case of the manifold $\mathbb{C} P^{1}$ relevant to $N=2$ theory. To see this, let us consider the following partial gauge field, $\mathscr{A}_{s}, s=1,2,3$, which defines a holomorphic bundle over $Q$ with $\mathbb{C}^{6}$ as a fibre:

$$
\begin{aligned}
& \mathscr{A}_{1 B}^{A}=2\left[\frac{p^{i} p^{j} \bar{p}_{(k} \bar{v}_{\ell)}}{|p|^{2}|v|^{2}}+\frac{p^{(i} v^{j} \bar{v}_{k} \bar{v}_{\ell}}{|v|^{4}}+\frac{p^{(i} \bar{u}^{j)} \bar{v}_{(k} u_{\ell)}}{|u|^{2}|v|^{2}}\right], \\
& \mathscr{A}_{2 B}^{A}=-2\left[\frac{p^{(i} v^{j)} \bar{p}_{(k} \bar{v}_{\ell)}}{|p|^{2}}+\frac{v^{i} v^{j} \bar{v}_{(k} u_{\ell)}}{|v|^{2}}+\frac{\bar{u}^{(i} v^{j)} u_{k} u_{\ell}}{|u|^{2}}\right], \\
& \mathscr{A}_{3 B}{ }^{A}=2\left[\frac{p^{i} p^{j} \bar{p}_{(k} u_{\ell)}}{|p|^{2}}+\frac{v^{(i} p^{j)} \bar{v}_{(k} u_{\ell)}}{|v|^{2}}+\frac{\bar{u}^{(i} p^{j)} u_{k} u_{\ell}}{|u|^{2}}\right] .
\end{aligned}
$$

Here $A=(i, j)=(j, i), B=(k, \ell)=(\ell, k), v^{i} \equiv\left(\bar{v}_{i}\right)^{*}=|p|^{-2} \varepsilon^{i j k} \bar{p}_{j} u_{k},|v|^{2}=|u|^{2} /|p|^{2}$. The functions $\mathscr{A}_{s B}^{A}$ in (11.1) are the matrix elements of the $6 \times 6$ matrix valued fields $\mathscr{A}_{s}$. 
One can verify that the six-dimensional holomorphic bundle corresponding to the partial gauge field (11.1) is topologically trivial, but non-trivial holomorphically. Moreover, it can be shown that every bundle sufficiently close to that one is holomorphically non-trivial as well.

The above discussion seems to imply that beyond perturbation theory the superfield formulation of $N=3$ theory on isotwistor space may be not equivalent to the usual Minkowski space formulation. We hope to return to this issue in a future publication.

Acknowledgements. We are grateful to A. S. Galperin, G. M. Henkin, R. E. Kallosh, Yu. I. Manin, V. I. Ogievetsky, I. B. Penkov, V. P. Palamodov, and A. A. Voronov for helpful discussions.

\section{References}

1. Galperin, A., Ivanov, E., Kalitzin, S., Ogievetsky, V., Sokatchev, E.: Unconstrained $N=2$ matter, Yang-Mills, and supergravity theories in harmonic superspace. Class. Quantum Grav. 1, 469 (1984)

2. Galperin, A., Ivanonv, E., Kalitzin, S., Ogievetsky, V., Sokatchev, E.: Unconstrained off-shell $N=3$ supersymmetric Yang-Mills theory. Class. Quantum Grav. 2, 155 (1985)

3. Ward, R.S.: On self-dual gauge fields. Phys. Lett. 61 A, 81-82 (1977)

4. Rosly, A.A.: Super Yang-Mills constraints as inegrability conditions. In: Group theoretical methods in physics. Proc. Int. Seminar, Zvenigorod 1982, Markov, M.A., (ed.) Moscow: Nauka 1, 263-268 (1983)

5. Penrose, R.: Nonlinear gravitons and curved twistor theory. Gen. Relativ. Gravitation 7, 3152 (1976)

6. Witten, E.: An interpretation of classical Yang-Mills fields. Phys. Lett. 77 B, 394-398 (1978)

7. Green, P.S., Isenberg, J., Yasskin, P.B.: Non-self-dual gauge fields. Phys. Lett. 78 B, 462-464 (1978)

8. Grimm, R., Sohnius, M., Wess, J.: Extended supersymmetry and gauge theories. Nucl. Phys. B 133, 275-284 (1978)

9. Deser, S., Jackiw, R., Templeton, S.: Three dimensional massive gauge theories. Phys. Rev. Lett. 48, 975-978 (1982)

10. Fayet, P.: Fermi-Bose hypersymmetry. Nucl. Phys. B 113, 135-155 (1976)

Sohnius, M.F.: Supersymmetry and central charges. Nucl. Phys. B 138, 109-121 (1978)

11. Malgrange, B.: Lectures on the theory of several complex variables. Bombay 1958

Wells, R.O.: Differential analysis on complex manifolds. Englewood Cliffs, N.J.: Prentice Hall 1973

Griffiths, P., Harris, J.: Principles of algebraic geometry. New York: Wiley 1978

12. Henkin, G.M.: Representation of Yang-Mills equations in the form of Cauchy-Riemann equations in the twistor space. Dokl. Akad. Nauk USSR 255, 844-847 (1980); Tangential Cauchy-Riemann equations and Yang-Mills, Higgs, and Dirac fields. In: Proc. ICM, Warsaw 1983

Henkin, G.M., Manin, Yu.I.: The Yang-Mills-Dirac equations as the Cauchy-Riemann equations in the twistor space. Yad. Fiz. 35, 1610-1626 (1982)

13. Kodaira, K., Spencer, D.C.: On deformations of complex analytic structures. I-II. Ann. Math. 67, $326-466$ (1958);

Onishchik, A.L.: On deformations of fibre bundles. Dokl. Akad. Nauk USSR 161, 45-47 (1965)

14. Rosly, A.A.: Gauge fields in superspace and twistors. Class. Quantum Grav. 2, 693-699 (1985)

Communicated by Ya. G. Sinai

Received December 15, 1985 